\title{
Active-Damping Virtual Circuit Control for Grid-Tied Converters with Differential-Mode and Common-Mode Output Filters
}

\author{
Korawich Niyomsatian, Member, IEEE, Piet Vanassche, Member, IEEE, Johan Gyselinck, Member, IEEE, \\ and Ruth V. Sabariego
}

\begin{abstract}
This paper presents a virtual circuit control (VCC) method of designing a resonant-damping discrete-time controller for grid-tied voltage source converters with differential-mode (DM) and common-mode (CM) output filters. The method provides an intuitive way to specify the desired closed-loop behavior by means of a virtual reference circuit rather than abstract mathematical criteria such as closed-loop poles and weighting matrices. Therefore, the existing passive filter designs, which cannot be practically implemented due to excessive losses, and the well-established theory of filters can be exploited. The DM grid current and the CM capacitor voltage, which are the primary control objectives, inherit the main properties of their underlying virtual reference circuits, e.g. resonance damping and low-frequency behavior. On this account, to fortify the controller against grid impedance variations, a virtual circuit with a series resistor at the grid side is considered. Accordingly, the $\mathrm{CM}$ voltage and DM current controllers can be easily designed based on the low-frequency behavior of virtual circuits. The method can also be straightforwardly equipped with conventional controllers to enhance system performance, such as harmonic compensation. The simulation and experimental results verify the effectiveness of the DM and CM resonant damping and dynamic performance.
\end{abstract}

Index Terms-Converter control, virtual circuit, resonance damping, grid-tied converter, LCL filter, LC filter

\section{INTRODUCTION}

$\mathbf{F}$ OR a grid-tied voltage source converter (VSC), an $L C L$ filter is widely preferred as a differential-mode (DM) filter over an $L$ filter owing to higher superior harmonic rejection and more compact design. Moreover, it can be integrated with an $L C$ common-mode (CM) filter based on the so-called virtual-neutral configuration [?] to mitigate both the $\mathrm{CM}$ current injected into the grid and the CM high-frequency 6-step switched voltage on the DC bus with respect to earth [?], [?]. The simplest configuration, which is of interest in

Manuscript received April 25, 2019; revised July 27, 2019 and September 30, 2019; accepted November 15, 2019. This work was supported in part by the EU funded Marie Curie ITN ADvanced Electric Powertrain Technology (ADEPT) project (grant number 607361).

K. Niyomsatian and P. Vanassche are with National Instruments Leuven, De Vunt 5, 3220 Holsbeek, Belgium. (e-mail: korawich.niyomsatian@ni.com piet.vanassche@ni.com.)

J. Gyselinck is with the Department BEAMS, Université Libre de Bruxelles, CP165/52 - Av. F.D. Roosevelt 50 - 1050 Brussels, Belgium. (e-mail: johan.gyselinck@ulb.ac.be)

R.V. Sabariego is with the Department of Electrical Engineering (ESAT), KU Leuven, Kasteelpark Arenberg 10, 3001 Leuven-Heverlee, Belgium and with EnergyVille, Thor Park 8310, 3600 Genk, Belgium. (e-mail: ruth.sabariego@kuleuven.be) this paper, is shown in Fig. 1. However, this filter inherently presents an $L C L$ resonance in the DM circuit (Fig. 1b) and an additional $L C$ resonance in the $\mathrm{CM}$ circuit (Fig. 1c) [?], [?].

These resonances can be deliberately alleviated by passive damping (PD) or active damping (AD) methods. This paper focuses on the latter by employing proper control strategies. In general, the $\mathrm{AD}$ methods supplement current/voltage controllers. The popular methods rely on the feedback of filter state variables, e.g. the feedback of the filter capacitor current or its equivalence [?], [?] and grid current [?]. However, these AD methods only stabilize the system but do not allow the dynamic of the whole system to be adjusted.

Since the linearized average model of VSCs with output filters is sufficiently accurate [?], various model-based linear control techniques are applicable and widely adopted to fully manipulate the system dynamic, i.e. the resonance damping and the current/voltage control. Since system poles and zeros determine linear-system dynamics, direct pole placement is a straightforward technique [?], [?], [?], [?], [?]. However, choosing proper locations of closed-loop poles and zeros is not a trivial task. Some simple rules of thumb which relate performance and robustness indices to pole/zero locations can be formulated only for low-order systems. Thus, a low-order approximation can be required for high-order systems e.g. using dominant poles at the cost of slower systems [?], [?], [?]. Still, the general relation remains an open challenge [?], which have been tackled by various numerical methods (see [?] and the references therein).

Disregarding closed-loop pole locations, the digital dead-beat control moves all the closed-loop poles to the origin yielding the fastest response with the loss of high susceptibility to model mismatches [?], [?]. Rather than stumbling upon the well-chosen closed-loop poles, the steady-state optimal linear quadratic (LQ) control finds a proper set of poles and a corresponding state-feedback gain based on the minimization of a cost function [?]. However, the cost function is weakly connected to the filter parameters and some desired performance specifications such as transient response and robustness [?]. In addition, more sophisticated model-based controls have been applied, e.g. model predictive control [?], adaptive control [?] and sliding-mode control [?]. Nevertheless, they normally result in complicated nonlinear closed-loop systems.

The original contribution of this paper is a new method of designing an AD-based discrete-time current controller based 


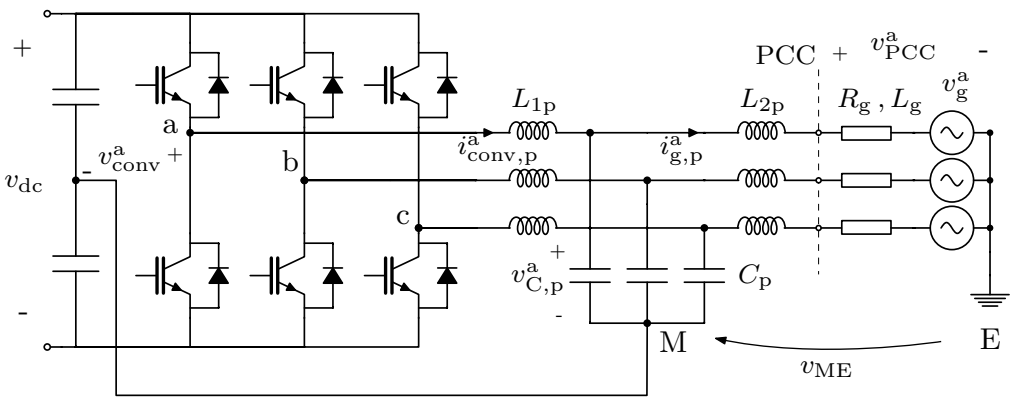

(a) Grid-tied converter with $L C L$ filter

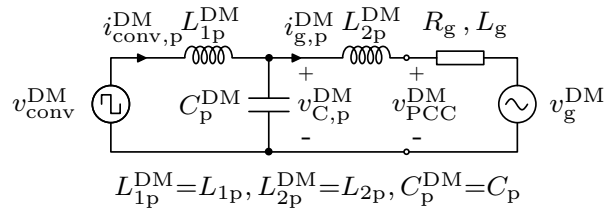

(b) Per-phase differential mode circuit

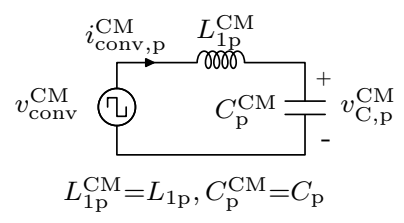

(c) Simplified common mode circuit

Fig. 1. Grid-tied converter with $L C L$ filter considered in this work. The filter circuit is decomposed into (a) DM and (b) CM circuits through the power-invariant Clarke transformation.

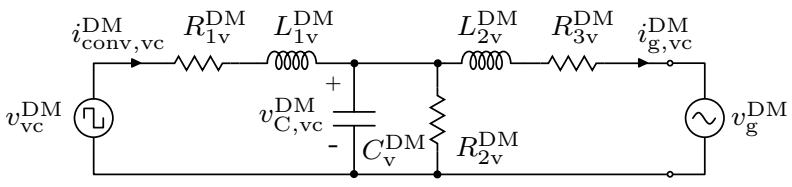

(a) $3^{\text {rd }}$-order DM virtual circuit

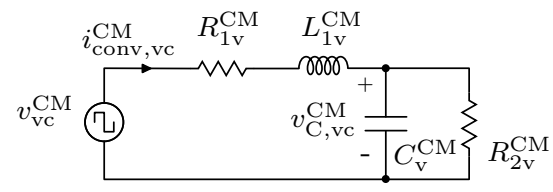

(b) $2^{\text {nd }}$-order $\mathrm{CM}$ virtual circuit

Fig. 2. Virtual reference circuits considered in this paper.

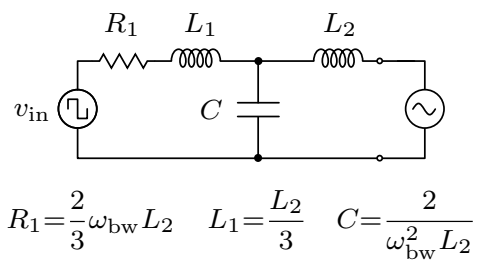

(a) $3^{\text {rd }}$-order circuit

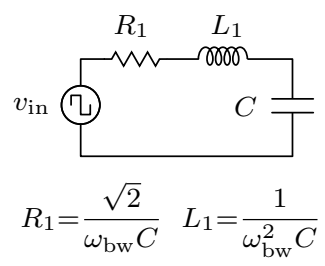

(b) $2^{\text {nd }}$-order circuit
Fig. 3. Butterworth-filter circuits.

on circuit viewpoint termed Virtual Circuit Control (VCC). Contrary to those conventional ways of only emulating some certain elements or impedances [?], [?], [?], the VCC method aims to mimic the behavior and inherit the properties of a desired reference circuit in Fig. 2. This general configuration of reference circuit is of the same order as the physical circuit (Fig. 1), but possesses the highest damping capability. This reference circuit does not physically exist and is therefore referred to as virtual circuit. The main benefits of this method are summarized as follows:

- The virtual circuit (Fig. 2) is designed as a formal but intuitive way for an electrical engineer to specify the desired closed-loop system behavior alternative to abstract mathematical criteria such as closed-loop poles or weighting matrices in the LQ control. For instance, it is difficult to intuitively adjust the desired closed-loop poles locations to counteract grid impedance variations, whereas the VCC method suggests an intuitive way to fortify a controller by using a virtual circuit with a resistor at the grid side $\left(R_{3 \mathrm{v}}^{\mathrm{DM}}\right.$ in Fig. 2a).

- The VCC method exploits the existing passive filter design, which cannot be practically realized due to excessive losses, and the well-established theory of filter synthesis [?]. For example, the Butterworth filters (Fig. 3) possess a well-damped property, which can be readily selected for a desired bandwidth (3-dB cut-off frequency, $\left.\omega_{\mathrm{bw}}=2 \pi f_{\mathrm{bw}}\right)$.

- The VCC method facilitates the design of current/voltage controllers and can be easily equipped with conventional current controllers.

This paper begins with the motivation of the VCC method in continuous-time (CT) controlled systems in Section II. Then, the VCC method is developed in the discrete-time (DT) domain for sampled-data systems in Section III. The introduction of an observer for compensating inherent digital delays and the integration of DM and CM controllers, and the auxiliary controllers are explained in Section IV. Three notable design examples of virtual circuits are illustrated in Section V. The simulation and experimental results are presented in Section VI.

\section{Motivation OF VCC METHOD BASED ON CONTINUOUS-TIME SYSTEMS}

Motivated by the idea of the model following control, the VCC method builds on two internal models: an uncontrolled physical circuit in Fig. 1 and a virtual reference circuit or so-called virtual circuit in Fig. 2. The former normally possesses some undesired behaviors, e.g. resonances and slow dynamics, whereas the latter expresses the desired behavior. Hereon, the subscripts ' $p$ ' and ' $v c$ ' distinguish the quantities of the 'physical' and 'virtual' circuits respectively.

\section{A. Continuous-Time Circuit Models}

The considered three-phase physical circuit in Fig. 1a can be modeled with a DM $L C L$ (per-phase) and a CM 
$L C$ equivalent circuits in Figs. 1b\&c respectively, and so is the virtual circuit counterpart of each (Figs. 2a\&b). These equivalent circuits are represented by linear average state-space models in the stationary coordinates. According to Fig. 1 and knowing the voltage at the point of common coupling (PCC) $v_{\mathrm{PCC}}$, the DM and CM physical circuits can be generally modeled by

$$
\dot{\mathbf{x}}_{\mathrm{p}}=\mathbf{A}_{\mathrm{p}} \mathbf{x}_{\mathrm{p}}+\mathbf{B}_{\mathrm{i}, \mathrm{p}} v_{\text {conv }}+\mathbf{B}_{\mathrm{o}, \mathrm{p}} v_{\mathrm{PCC}}
$$

where hereon superscripts 'DM' and 'CM' denote the quantities associated with the DM and CM circuits respectively. From Figs. 1b\&c, the system matrices of the DM and $\mathrm{CM}$ physical circuits are

$$
\begin{aligned}
& \mathbf{x}_{\mathrm{p}}^{\mathrm{DM}}=\left[\begin{array}{c}
v_{\mathrm{C}, \mathrm{p}}^{\mathrm{DM}} \\
i_{\mathrm{conv}, \mathrm{p}}^{\mathrm{DM}} \\
i_{\mathrm{g}, \mathrm{p}}^{\mathrm{DM}}
\end{array}\right], \quad \mathbf{A}_{\mathrm{p}}^{\mathrm{DM}}=\left[\begin{array}{ccc}
0 & \frac{1}{C_{\mathrm{p}}^{\mathrm{DM}}} & -\frac{1}{C_{\mathrm{p}}^{\mathrm{DM}}} \\
-\frac{1}{L_{1 \mathrm{p}}^{\mathrm{DM}}} & 0 & 0 \\
\frac{1}{L_{2 \mathrm{p}}^{\mathrm{DM}}} & 0 & 0
\end{array}\right], \\
& \mathbf{B}_{\mathrm{i}, \mathrm{p}}^{\mathrm{DM}}=\left[\begin{array}{c}
0 \\
\frac{1}{L_{1 \mathrm{p}}^{\mathrm{DM}}} \\
0
\end{array}\right], \quad \mathbf{B}_{\mathrm{O}, \mathrm{p}}^{\mathrm{DM}}=\left[\begin{array}{c}
0 \\
0 \\
-\frac{1}{L_{2 \mathrm{p}}^{\mathrm{DM}}}
\end{array}\right] \text {, } \\
& \mathbf{x}_{\mathrm{p}}^{\mathrm{CM}}=\left[\begin{array}{c}
v_{\mathrm{C}, \mathrm{p}}^{\mathrm{CM}} \\
i_{\text {conv }, \mathrm{p}}^{\mathrm{CM}}
\end{array}\right], \quad \mathbf{A}_{\mathrm{p}}^{\mathrm{CM}}=\left[\begin{array}{cc}
0 & \frac{1}{C_{\mathrm{p}}^{\mathrm{CM}}} \\
-\frac{1}{L_{1 \mathrm{p}}^{\mathrm{CM}}} & 0
\end{array}\right], \\
& \mathbf{B}_{\mathrm{i}, \mathrm{p}}^{\mathrm{CM}}=\left[\begin{array}{c}
0 \\
\frac{1}{L_{1 \mathrm{p}}^{\mathrm{CM}}}
\end{array}\right], \quad \mathbf{B}_{\mathrm{O}, \mathrm{p}}^{\mathrm{CM}}=\left[\begin{array}{l}
0 \\
0
\end{array}\right] .
\end{aligned}
$$

In contrast to the physical circuit, the DM and CM virtual circuits in Figs. 2a\&b are not coupled and can be independently selected. They are modeled by

$$
\dot{\mathbf{x}}_{\mathrm{vc}}=\mathbf{A}_{\mathrm{vc}} \mathbf{x}_{\mathrm{vc}}+\mathbf{B}_{\mathrm{i}, \mathrm{vc}} v_{\mathrm{vc}}+\mathbf{B}_{\mathrm{o}, \mathrm{vc}} v_{\mathrm{PCC}}
$$

where

$$
\begin{aligned}
\mathbf{x}_{\mathrm{vc}}^{\mathrm{DM}}=\left[\begin{array}{c}
v_{\mathrm{C}, \mathrm{vc}}^{\mathrm{DM}} \\
i_{\mathrm{conv}, \mathrm{vc}}^{\mathrm{DM}} \\
i_{\mathrm{g}, \mathrm{vc}}^{\mathrm{DM}}
\end{array}\right], & \mathbf{A}_{\mathrm{vc}}^{\mathrm{DM}}=\left[\begin{array}{ccc}
-\frac{1}{R_{2 \mathrm{v}}^{\mathrm{DM}} C_{\mathrm{v}}^{\mathrm{DM}}} & \frac{1}{C_{\mathrm{v}}^{\mathrm{DM}}} & -\frac{1}{C_{\mathrm{v}}^{\mathrm{DM}}} \\
-\frac{1}{L_{1 \mathrm{v}}^{\mathrm{DM}}} & -\frac{R_{1 \mathrm{v}}^{\mathrm{DM}}}{L_{1 \mathrm{v}}^{\mathrm{DM}}} & 0 \\
\frac{1}{L_{2 \mathrm{v}}^{\mathrm{DM}}} & 0 & -\frac{R_{3 \mathrm{v}}^{\mathrm{DM}}}{L_{2 \mathrm{v}}^{\mathrm{DM}}}
\end{array}\right] \\
\mathbf{B}_{\mathrm{i}, \mathrm{vc}}^{\mathrm{DM}}=\left[\begin{array}{c}
0 \\
\frac{1}{L_{1 \mathrm{v}}^{\mathrm{DM}}} \\
0
\end{array}\right], & \mathbf{B}_{\mathrm{O}, \mathrm{vc}}^{\mathrm{DM}}=\left[\begin{array}{c}
0 \\
0 \\
-\frac{1}{L_{2 \mathrm{v}}^{\mathrm{DM}}}
\end{array}\right], \\
\mathbf{x}_{\mathrm{vc}}^{\mathrm{CM}}=\left[\begin{array}{c}
v_{\mathrm{C}, \mathrm{vc}}^{\mathrm{CM}} \\
i_{\mathrm{conv}, \mathrm{vc}}^{\mathrm{CM}}
\end{array}\right], & \mathbf{A}_{\mathrm{vc}}^{\mathrm{CM}}=\left[\begin{array}{cc}
-\frac{1}{R_{2 \mathrm{v}}^{\mathrm{CM}} C_{\mathrm{v}}^{\mathrm{CM}}} & \frac{1}{C_{\mathrm{v}}^{\mathrm{CM}}} \\
-\frac{1}{L_{1 \mathrm{v}}^{\mathrm{CM}}} & -\frac{R_{1 \mathrm{v}}^{\mathrm{CM}}}{L_{1 \mathrm{v}}^{\mathrm{CM}}}
\end{array}\right], \\
\mathbf{B}_{\mathrm{i}, \mathrm{vc}}^{\mathrm{CM}}=\left[\begin{array}{c}
0 \\
\frac{1}{L_{1 \mathrm{v}}^{\mathrm{CM}}}
\end{array}\right], & \mathbf{B}_{\mathrm{O}, \mathrm{vc}}^{\mathrm{CM}}=\left[\begin{array}{l}
0 \\
0
\end{array}\right] .
\end{aligned}
$$

\section{B. Transformations between Physical and Virtual Circuits}

The physical and virtual circuits must be compared on the same basis or coordinates [?]. In contrast to a well-known trajectory generation and tracking problem, a state generated from the virtual circuit is not necessarily a feasible trajectory satisfying the dynamic of the physical circuit [?]. For example, considering a DM virtual circuit in Fig. $2 \mathrm{a}$ with only a parallel resistor $R_{2 \mathrm{v}}^{\mathrm{DM}}$, the same grid currents $\left(i_{\mathrm{g}, \mathrm{p}}^{\mathrm{DM}}=i_{\mathrm{g}, \mathrm{vc}}^{\mathrm{DM}}\right)$ and capacitor voltages $\left(v_{\mathrm{C}, \mathrm{p}}^{\mathrm{DM}}=v_{\mathrm{C}, \mathrm{vc}}^{\mathrm{DM}}\right)$ of both physical and virtual circuits result in unequal converter currents $\left(i_{\text {conv }, \mathrm{p}}^{\mathrm{DM}} \neq i_{\mathrm{conv}, \mathrm{vc}}^{\mathrm{DM}}=i_{\mathrm{g}, \mathrm{vc}}^{\mathrm{DM}}+v_{\mathrm{C}, \mathrm{vc}}^{\mathrm{DM}} / R_{2 \mathrm{v}}^{\mathrm{DM}}\right)$. This invokes the requirement of a linear similarity transformation $\mathbf{T}_{\mathrm{c}}$, namely $\mathbf{T}_{\mathrm{c}}^{\mathrm{DM}} \in \mathbb{R}^{3 \times 3}$ and $\mathbf{T}_{\mathrm{c}}^{\mathrm{CM}} \in \mathbb{R}^{2 \times 2}$, to respectively map the behaviors (states) of DM/CM physical and virtual circuits on each other or

$$
\mathbf{x}_{\mathrm{p}}^{\mathrm{DM}}(t)=\mathbf{T}_{\mathrm{c}}^{\mathrm{DM}} \mathbf{x}_{\mathrm{vc}}^{\mathrm{DM}}(t), \quad \mathbf{x}_{\mathrm{p}}^{\mathrm{CM}}(t)=\mathbf{T}_{\mathrm{c}}^{\mathrm{CM}} \mathbf{x}_{\mathrm{vc}}^{\mathrm{CM}}(t) .
$$

\section{Continuous-Time State Feedback Control Law}

Assuming a control law for the physical circuit as a static state feedback, i.e. $v_{\text {conv }}=-\mathbf{L}_{\mathrm{xc}} \mathbf{x}_{\mathrm{p}}+L_{\mathrm{uc}} v_{\mathrm{vc}}+L_{\mathrm{oc}} v_{\mathrm{PCC}}$, with constant gains $\mathbf{L}_{\mathrm{xc}}, L_{\mathrm{uc}}$ and $L_{\mathrm{oc}}$, the controlled physical circuit becomes

$\dot{\mathbf{x}}_{\mathrm{p}}=\left(\mathbf{A}_{\mathrm{p}}-\mathbf{L}_{\mathrm{xc}} \mathbf{B}_{\mathrm{i}, \mathrm{p}}\right) \mathbf{x}_{\mathrm{p}}+L_{\mathrm{uc}} \mathbf{B}_{\mathrm{i}, \mathrm{p}} v_{\mathrm{vc}}+\left(L_{\mathrm{oc}} \mathbf{B}_{\mathrm{i}, \mathrm{p}}+\mathbf{B}_{\mathrm{o}, \mathrm{p}}\right) v_{\mathrm{PCC}}$

Based on (3), the controlled physical circuit in (4) and the virtual circuit in (2) are equivalent under transformation $\mathbf{T}_{\mathrm{c}}$, i.e. $\mathbf{T}_{\mathrm{c}}^{\mathrm{DM}}$ and $\mathbf{T}_{\mathrm{c}}^{\mathrm{CM}}$ for $\mathrm{DM}$ and $\mathrm{CM}$ systems respectively, if and only if the following conditions hold:

$$
\begin{gathered}
\left(\mathbf{A}_{\mathrm{p}}-\mathbf{L}_{\mathrm{xc}} \mathbf{B}_{\mathrm{i}, \mathrm{p}}\right) \mathbf{T}_{\mathrm{c}}=\mathbf{T}_{\mathrm{c}} \mathbf{A}_{\mathrm{vc}} \\
L_{\mathrm{uc}} \mathbf{B}_{\mathrm{i}, \mathrm{p}}=\mathbf{T}_{\mathrm{c}} \mathbf{B}_{\mathrm{i}, \mathrm{vc}} \\
L_{\mathrm{oc}} \mathbf{B}_{\mathrm{i}, \mathrm{p}}+\mathbf{B}_{\mathrm{o}, \mathrm{p}}=\mathbf{T}_{\mathrm{c}} \mathbf{B}_{\mathrm{o}, \mathrm{vc}} .
\end{gathered}
$$

Since both the physical and virtual circuits are controllable from the control input, but not from the PCC voltage, three conditions (5)-(7) cannot always be simultaneously satisfied. Instead, the PCC voltage is treated as a disturbance [?].

Considering only (5) and (6), there exists a static feedback gain $\mathbf{L}_{\mathrm{xc}}$ such that $\mathbf{A}_{\mathrm{p}}-\mathbf{L}_{\mathrm{xc}} \mathbf{B}_{\mathrm{i}, \mathrm{p}}$ and $\mathbf{A}_{\mathrm{vc}}$ possess an identical set of eigenvalues. In other words, the homogeneous Sylvester equation in (5) as well as (6) always has a non-trivial solution $\mathbf{T}_{\mathrm{c}}, \mathbf{L}_{\mathrm{xc}}$ and $L_{\mathrm{uc}}$. The gains $L_{\mathrm{uc}}$ and $L_{\mathrm{oc}}$ can be chosen to match the low-frequency response of the physical and virtual circuits. The detailed derivation of the solution is given in Appendix, and only the insightful results of transformations $\mathbf{T}_{\mathrm{c}}^{\mathrm{DM}}$ and $\mathbf{T}_{\mathrm{c}}^{\mathrm{CM}}$ are presented here in (8) on the bottom of the next page.

From (3), the primary control quantities, namely the DM grid current $i_{\mathrm{g}, \mathrm{p}}^{\mathrm{DM}}$ and the CM capacitor voltage $v_{\mathrm{C}, \mathrm{p}}^{\mathrm{CM}}$ of the controlled physical circuit in Fig. 1b\&c, can be written as a linear combination of virtual states $\mathbf{x}_{\mathrm{vc}}^{\mathrm{DM}}$ and $\mathbf{x}_{\mathrm{vc}}^{\mathrm{CM}}$. In other words, $i_{\mathrm{g}, \mathrm{p}}^{\mathrm{DM}}$ linearly depends on $\mathrm{x}_{\mathrm{vc}}^{\mathrm{DM}}$ according to the last-row entries of $\mathbf{T}_{\mathrm{c}}^{\mathrm{DM}}$, i.e. $\kappa_{r, \mathrm{c}}^{\mathrm{DM}}=\left[\mathbf{T}_{\mathrm{c}}^{\mathrm{DM}}\right]_{3 r}, r \in\{1,2,3\} ; i_{\mathrm{g}, \mathrm{p}}^{\mathrm{DM}}=$ $\kappa_{1, \mathrm{c}}^{\mathrm{DM}} v_{\mathrm{C}, \mathrm{vc}}^{\mathrm{DM}}+\kappa_{2, \mathrm{c}}^{\mathrm{DM}} i_{\mathrm{conv}, \mathrm{vc}}^{\mathrm{DM}}+\kappa_{3, \mathrm{c}}^{\mathrm{DM}} i_{\mathrm{g}, \mathrm{vc}}^{\mathrm{DM}}$. In addition, $v_{\mathrm{C}, \mathrm{p}}^{\mathrm{CM}}$ linearly depends on $\mathbf{x}_{\mathrm{vc}}^{\mathrm{CM}}$ according to the first-row entries of $\mathbf{T}_{\mathrm{c}}^{\mathrm{CM}}$, i.e. $\kappa_{s, \mathrm{c}}^{\mathrm{CM}}=\left[\mathbf{T}_{\mathrm{c}}^{\mathrm{CM}}\right]_{1 s}, s \in\{1,2\} ; v_{\mathrm{C}, \mathrm{p}}^{\mathrm{CM}}=\kappa_{1, \mathrm{c}}^{\mathrm{CM}} v_{\mathrm{C}, \mathrm{vc}}^{\mathrm{CM}}+\kappa_{2, \mathrm{c}}^{\mathrm{CM}} i_{\mathrm{conv}, \mathrm{vc}}^{\mathrm{CM}}$. From (8), only $\kappa_{3, \mathrm{c}}^{\mathrm{DM}}, \kappa_{1, \mathrm{c}}^{\mathrm{CM}}=1$, while the rest are zero; hence $i_{\mathrm{g}, \mathrm{p}}^{\mathrm{DM}}$ and $v_{\mathrm{C}, \mathrm{p}}^{\mathrm{CM}}$ perfectly match their virtual circuit counterparts or

$$
i_{\mathrm{g}, \mathrm{p}}^{\mathrm{DM}}=i_{\mathrm{g}, \mathrm{vc}}^{\mathrm{DM}}, v_{\mathrm{C}, \mathrm{p}}^{\mathrm{CM}}=v_{\mathrm{C}, \mathrm{vc}}^{\mathrm{CM}} .
$$

In other words, the design of the control law boils down to the design of the virtual circuit, which is more intuitive for electrical engineers. 
Nevertheless, it can be expected that the virtual circuit should not be designed arbitrarily. The good virtual circuits should resemble the physical circuit (cf. Figs. 1\&2). Otherwise, it can cause less robustness and aggressive control actions for tracking virtual circuit behavior. This could be roughly indicated by the sensitivity of the physical state $\mathbf{x}_{\mathrm{p}}$ to the desired virtual state $\mathbf{x}_{\mathrm{vc}}$ or the condition number of transformation $\mathbf{T}_{\mathrm{c}}$ [?]. If the virtual circuit differs from the physical one only in the elements next to converters, i.e. $\left\{R_{1 \mathrm{v}}^{\mathrm{DM}}, L_{1 \mathrm{v}}^{\mathrm{DM}}\right\}$ and $\left\{R_{1 \mathrm{v}}^{\mathrm{CM}}, L_{1 \mathrm{v}}^{\mathrm{CM}}\right\}, \mathbf{T}_{\mathrm{c}}^{\mathrm{DM}}$ and $\mathbf{T}_{\mathrm{c}}^{\mathrm{CM}}$ in (8) become identity matrices yielding the lowest condition number of unity. Equivalently, these circuit configurations are the ones of the closest stable systems to marginally stable $L C L$ and $L C$ systems [?]. This suggests the design of a virtual circuit by modifying only $\left\{R_{1 \mathrm{v}}^{\mathrm{DM}}, L_{1 \mathrm{v}}^{\mathrm{DM}}\right\}$ and $\left\{R_{1 \mathrm{v}}^{\mathrm{CM}}, L_{1 \mathrm{v}}^{\mathrm{CM}}\right\}$.

\section{Proposed VCC Method For SAMPLED-DATA SYSTEM}

In practice, a digital control law is considered instead of an analog counterpart. Herein, the zero-order-hold $(\mathrm{ZOH})$ discretization with sampling interval $T_{\mathrm{s}}$ is considered to account for half a sample delay due to digital pulsewidth modulators (PWM) [?]. The $\mathrm{ZOH}$ equivalent model of the $\mathrm{DM} / \mathrm{CM}$ physical and virtual circuits (with superscripts 'DM' and 'CM' and subscripts ' $\mathrm{p}$ ' and ' $\mathrm{vc}$ ' where necessary) in (1) and (2) are in the form

$$
\mathbf{x}_{k+1}=\mathbf{F x}_{k}+\mathbf{G}_{\mathrm{i}} v_{\mathrm{i}, k}+\mathbf{G}_{\mathrm{o}} v_{\mathrm{PCC}, k}
$$

where $\mathbf{F}=\mathrm{e}^{T_{\mathrm{s}} \mathbf{A}}, \quad \mathbf{G}_{\mathrm{i}}=\int_{0}^{T_{\mathrm{s}}} \mathrm{e}^{\tau \mathbf{A}} \mathbf{B}_{\mathrm{i}} \mathrm{d} \tau, \quad \mathbf{G}_{\mathrm{o}}=\int_{0}^{T_{\mathrm{s}}} \mathrm{e}^{\tau \mathbf{A}} \mathbf{B}_{\mathrm{o}} \mathrm{d} \tau$, $v_{\mathrm{i}}$ is $v_{\text {conv }}$ and $v_{\mathrm{vc}}$ for the physical and virtual circuits respectively, and subscript $k$ denotes the value at the time instant $t=k T_{\mathrm{S}}$.

Analogous to (3), a transformation $\mathbf{T}_{\mathrm{d}}$, i.e. $\mathbf{T}_{\mathrm{d}}^{\mathrm{DM}}$ and $\mathbf{T}_{\mathrm{d}}^{\mathrm{CM}}$ for DM and CM systems, is introduced to map the sampled physical state $\mathbf{x}_{\mathrm{p}, k}$ and virtual state $\mathbf{x}_{\mathrm{vc}, k}$ on each other. The control action $v_{\text {conv }, k}$ for the control interval $\left[k T_{\mathrm{s}},(k+1) T_{\mathrm{s}}\right]$ is derived from the condition that the behavior of a quantity of interest mimics that of the virtual circuit under transformation $\mathbf{T}_{\mathrm{d}}$ or

$$
v_{\text {conv }, k}=\underset{v_{\text {conv }, k}, \mathbf{T}_{\mathrm{d}}}{\arg \min _{k T_{\mathrm{s}}}^{(k+1) T_{\mathrm{s}}}}\left\|w(t) \mathbf{P}\left[\mathbf{x}_{\mathrm{p}}\left(v_{\mathrm{conv}, k}\right)-\mathbf{T}_{\mathrm{d}} \mathbf{x}_{\mathrm{vc}}\right]\right\|^{2} \mathrm{~d} t
$$

where $w(t)$ is a weighting function and $\mathbf{P}$ is a constant matrix choosing the quantity of comparison. If $w(t)=1$, formulation (11) becomes a least-square problem as demonstrated in [?], [?].
As a main interest of this paper, considering $\mathbf{P}$ an identity matrix and $w(t)$ a Dirac-delta function reading

$$
w(t)=\delta\left(t-(k+1) T_{\mathrm{s}}\right),
$$

formulation (11) becomes a collocation problem [?]. In other words, the states of the physical and virtual circuits are identical at sampling instants under transformation $\mathbf{T}_{\mathrm{d}}$. Mathematically, the two circuits share the same eigenvalues. Therefore, the solutions to (11) is a static feedback control law of the form

$$
v_{\text {conv }, k}=\mathbf{L}_{\mathrm{x}} \mathbf{x}_{\mathrm{p}, k}+L_{\mathrm{u}} v_{\mathrm{vc}, k}+L_{\mathrm{o}} v_{\mathrm{PCC}, k}
$$

with constant gains $\mathbf{L}_{\mathrm{x}}, L_{\mathrm{u}}$ and $L_{\mathrm{o}}$. The gains can be precomputed and are derived in Appendix. Although the final result is mathematically identical to the pole-placement problem, the desired closed-loop poles are selected through a design of a virtual circuit instead.

For sampled-data systems, the entries of transformations $\mathbf{T}_{\mathrm{d}}^{\mathrm{DM}}$ and $\mathbf{T}_{\mathrm{d}}^{\mathrm{CM}}$ depend on the sampling frequency $f_{\mathrm{s}}=1 / T_{\mathrm{s}}$ in contrast to (8) and (9) in the CT domain. Therefore, the sampled physical DM grid current $i_{\mathrm{g}, \mathrm{p}, k}^{\mathrm{DM}}$ and $\mathrm{CM}$ capacitor voltage $v_{\mathrm{C}, \mathrm{p}, k}^{\mathrm{CM}}$ do not perfectly track the virtual counterparts $i_{\mathrm{g}, \mathrm{vc}, k}^{\mathrm{DM}}$ and $v_{\mathrm{C}, \mathrm{vc}, k}^{\mathrm{CM}}$. They are instead the linear combinations of the virtual states $\mathbf{x}_{\mathrm{vc}}^{\mathrm{DM}}$ and $\mathbf{x}_{\mathrm{vc}}^{\mathrm{CM}}$ according to linear transformations $\mathbf{T}_{\mathrm{d}}^{\mathrm{DM}}$ and $\mathbf{T}_{\mathrm{d}}^{\mathrm{CM}}$. Normalizing the quantities in per unit $(\mathrm{pu})$, we obtain

$$
\begin{aligned}
i_{\mathrm{g}, k}^{\mathrm{DM}, \mathrm{pu}}= & \kappa_{1, \mathrm{~d}}^{\mathrm{DM}} v_{\mathrm{C}, \mathrm{vc}, k}^{\mathrm{DM}, \mathrm{pu}}+\kappa_{2, \mathrm{~d}}^{\mathrm{DM}} i_{\mathrm{conv}, \mathrm{vc}, k}^{\mathrm{DM}, \mathrm{pu}}+\kappa_{3, \mathrm{~d}}^{\mathrm{DM}} i_{\mathrm{g}, \mathrm{vc}, k}^{\mathrm{DM}, \mathrm{pu}} \\
& v_{\mathrm{C}, k}^{\mathrm{CM}, \mathrm{pu}}=\kappa_{1, \mathrm{~d}}^{\mathrm{CM}} v_{\mathrm{C}, \mathrm{vc}, k}^{\mathrm{CM}, \mathrm{pu}}+\kappa_{2, \mathrm{~d}}^{\mathrm{CM}} i_{\text {conv }, \mathrm{vc}, k}^{\mathrm{CM}, \mathrm{pu}}
\end{aligned}
$$

where the coefficients $\kappa_{r, \mathrm{~d}}^{\mathrm{DM}}, r \in\{1,2,3\}$, and $\kappa_{s, \mathrm{~d}}^{\mathrm{CM}}, s \in\{1,2\}$, are the last-row and first-row entries of normalized $\mathbf{T}_{\mathrm{d}}^{\mathrm{DM}}$ and $\mathbf{T}_{\mathrm{d}}^{\mathrm{CM}}$ respectively.

Fig. 4 displays the coefficients $\kappa_{r, \mathrm{~d}}^{\mathrm{DM}}$ and $\kappa_{s, \mathrm{~d}}^{\mathrm{CM}}$ using the Butterworth virtual circuit in Fig. 3 for DM and CM virtual circuits with different bandwidth $f_{\mathrm{bw}}$ and the sampling frequency $f_{\mathrm{s}}=16 \mathrm{kHz}$. At low bandwidth relative to the sampling frequency $f_{\mathrm{bw}} / f_{\mathrm{s}}$, coefficients $\kappa_{r, \mathrm{~d}}^{\mathrm{DM}}$ and $\kappa_{s, \mathrm{~d}}^{\mathrm{CM}}$ converge to their CT counterparts $\kappa_{r, \mathrm{c}}^{\mathrm{DM}}$ and $\kappa_{s, \mathrm{c}}^{\mathrm{CM}} ; \kappa_{3, \mathrm{~d}}^{\mathrm{DM}} \rightarrow 1$ and $\kappa_{1, \mathrm{~d}}^{\mathrm{DM}}, \kappa_{2, \mathrm{~d}}^{\mathrm{DM}}, \kappa_{2, \mathrm{~d}}^{\mathrm{CM}} \rightarrow 0$ (Figs. $4 \mathrm{a} \& \mathrm{c}$ ). In other words, the relations (14) and (15) approach (9). Note that $\kappa_{1, \mathrm{~d}}^{\mathrm{CM}}$ remains around unity for the whole range of bandwidth frequency similar to $\kappa_{1, \mathrm{c}}^{\mathrm{CM}}$.

When $R_{2 \mathrm{v}}^{\mathrm{DM}}=10 R_{1 \mathrm{v}}^{\mathrm{DM}}$ and $R_{3 \mathrm{v}}^{\mathrm{DM}}=R_{1 \mathrm{v}}^{\mathrm{DM}}$ from Fig. $2 \mathrm{a}$ are included in the designed DM Butterworth circuit, the coefficients show more deviations particularly when the bandwidth frequency approaches the Nyquist frequency $\left(f_{\mathrm{bw}} / f_{\mathrm{s}}=0.5\right) \quad$ (see Fig. 4b). Still, the contribution of $i_{\mathrm{g}, \mathrm{vc}, k}^{\mathrm{DM}}$ in (14) is always dominant below $f_{\mathrm{bw}} / f_{\mathrm{s}}=0.3$, i.e.

$$
\mathbf{T}_{\mathrm{c}}^{\mathrm{DM}}=\left[\begin{array}{ccc}
L_{2 \mathrm{p}}^{\mathrm{DM}} & 0 & -\frac{L_{2 \mathrm{p}}^{\mathrm{DM}} R_{3 \mathrm{v}}^{\mathrm{DM}}}{L_{2 \mathrm{v}}^{\mathrm{DM}}} \\
-\frac{C_{\mathrm{p}}^{\mathrm{DM}} L_{2 \mathrm{p}}^{\mathrm{DM}}\left(C_{\mathrm{v}}^{\mathrm{DM}} R_{2 \mathrm{v}}^{\mathrm{DM}} R_{3 \mathrm{v}}^{\mathrm{DM}}+L_{2 \mathrm{v}}^{\mathrm{DM}}\right)}{C_{\mathrm{v}}^{\mathrm{DM}} L_{2 \mathrm{v}}^{\mathrm{DM}} R_{2 \mathrm{v}}^{\mathrm{DM}}} & \frac{C_{\mathrm{p}}^{\mathrm{DM}} L_{2 \mathrm{p}}^{\mathrm{DM}}}{C_{\mathrm{v}}^{\mathrm{DM}} L_{2 \mathrm{v}}^{\mathrm{DM}}} & 1+\frac{C_{\mathrm{p}}^{\mathrm{DM}} L_{2 \mathrm{p}}^{\mathrm{DM}} R_{3 \mathrm{v}}^{\mathrm{DM}}}{L_{2 \mathrm{v}}^{\mathrm{DM}}{ }^{2}}-\frac{C_{\mathrm{p}}^{\mathrm{DM}} L_{2 \mathrm{p}}^{\mathrm{DM}}}{C_{\mathrm{v}}^{\mathrm{DM}} L_{2 \mathrm{v}}^{\mathrm{DM}}}
\end{array}\right], \quad \mathbf{T}_{\mathrm{c}}^{\mathrm{DM}}=\left[\begin{array}{c}
1 \\
0
\end{array}\right.
$$




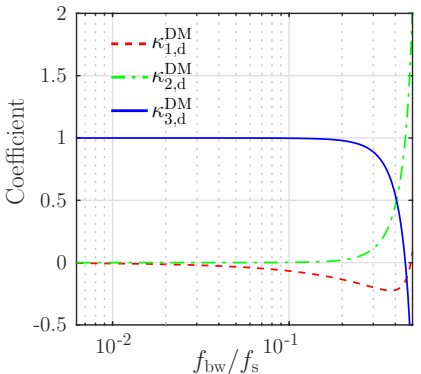

(a) Butterworth circuit in Fig. 3a $\left(R_{2 \mathrm{v}}^{\mathrm{DM}} \rightarrow \infty, R_{3 \mathrm{v}}^{\mathrm{DM}}=0\right)$

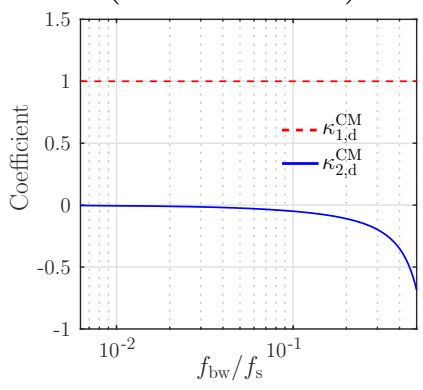

(c) Butterworth circuit in Fig. 3b $\left(R_{2 \mathrm{v}}^{\mathrm{CM}} \rightarrow \infty\right)$

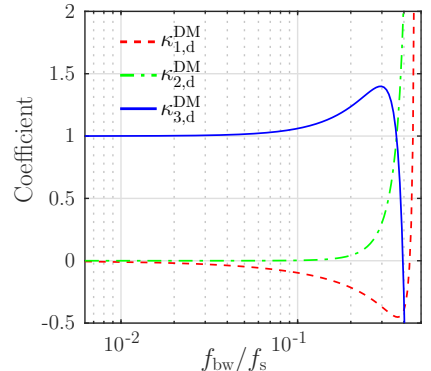

(b) Circuit in (a) with $R_{2 \mathrm{v}}^{\mathrm{DM}}=10 R_{1 \mathrm{v}}^{\mathrm{DM}}, R_{3 \mathrm{v}}^{\mathrm{DM}}=R_{1 \mathrm{v}}^{\mathrm{DM}}$

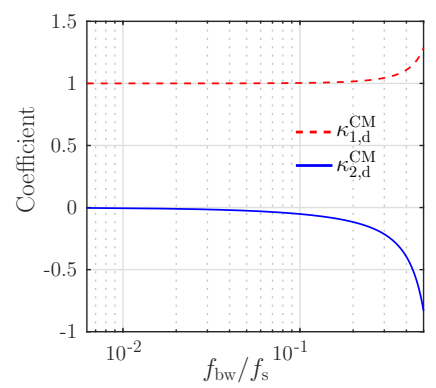

(d) Circuit in (c) with $R_{2 \mathrm{v}}^{\mathrm{CM}}=10 R_{1 \mathrm{v}}^{\mathrm{CM}}$
Fig. 4. Coefficients of linear dependence of DT VCC-controlled physical DM grid current and CM capacitor voltage on virtual states in per unit versus the bandwidth $f_{\mathrm{bw}}$ normalized with the sampling frequency $f_{\mathrm{s}}=16 \mathrm{kHz}$ : (a)-(b) $i_{\mathrm{g}, \mathrm{p}}^{\mathrm{DM}}$ on $\mathbf{x}_{\mathrm{vc}}^{\mathrm{DM}}$ from (14) and (c)-(d) $v_{\mathrm{C}, \mathrm{p}}^{\mathrm{CM}}$ on $\mathbf{x}_{\mathrm{vc}}^{\mathrm{CM}}$ from (15). The virtual circuit is designed based on the Butterworth circuits in Fig. 3 with $L_{2 \mathrm{v}}^{\mathrm{DM}}=L_{2 \mathrm{p}}^{\mathrm{DM}}$ and $C_{\mathrm{v}}^{\mathrm{CM}}=C_{\mathrm{p}}^{\mathrm{CM}}$. As an example, additional resistors are also added: (b) $R_{2 \mathrm{v}}^{\mathrm{DM}}$ and $R_{3 \mathrm{v}}^{\mathrm{DM}}$, and (d) $R_{2 \mathrm{v}}^{\mathrm{CM}}$.

$\kappa_{3, \mathrm{~d}}^{\mathrm{DM}} \gg \kappa_{1, \mathrm{~d}}^{\mathrm{DM}}, \kappa_{2, \mathrm{~d}}^{\mathrm{DM}}$. When $R_{2 \mathrm{v}}^{\mathrm{CM}}=10 R_{1 \mathrm{v}}^{\mathrm{CM}}$ is added in the designed CM Butterworth circuit, only the slight deviation of $\kappa_{1, \mathrm{~d}}^{\mathrm{CM}}$ from Fig. $4 \mathrm{c}$ can be observed close to the Nyquist frequency (Fig. 4d).

To summarize, this confirms that the design of a sampled-data controlled system can also boil down to the design of a virtual circuit if the circuit bandwidth is sufficiently low compared to the Nyquist frequency. Nevertheless, a careful consideration might be required when including a grid-side resistance $R_{3 \mathrm{v}}^{\mathrm{DM}}$.

\section{VCC Implementation AND MathematicAl Model}

This section elaborates the digital implementation of the VCC method. An observer is introduced to compensate the inherent computational delay of the converter control. The implementation is the same for both DM and CM circuits and thus the superscripts 'DM' and 'CM' are omitted. Subsequently, the combination of DM current control and CM voltage control using VCC method is explained. The optional auxiliary control can also be integrated. As a result, the simplified diagram of the whole system is depicted in Fig. 5.

\section{A. Delay Compensation and Observer}

Considering the computational delay (latency) of $N$ samples, the converter voltage $v_{\text {conv }}$ is applied $N$ samples after the computed converter voltage $v_{\text {conv }}^{\prime}$ is ready i.e. $v_{\text {conv }, k}=v_{\text {conv }, k-N}^{\prime}$. To compensate this delay, instead of (13), the computed converter voltage is thus calculated $N$-step ahead from

$$
v_{\text {conv }, k}^{\prime}=\mathbf{L}_{\mathrm{x}} \hat{\mathbf{x}}_{\mathrm{p}, k+N}+L_{\mathrm{u}} v_{\mathrm{vc}, k+N}+L_{\mathrm{o}} \hat{v}_{\mathrm{PCC}, k+N}
$$

where the hat ^ denotes an estimated quantity. For the VCC method to be effective and robust, $\hat{\mathbf{x}}_{\mathrm{p}, k+N}$ for (16) should then be accurately estimated ${ }^{1}$. Therefore, an observer together with a measurement is employed.

In this study, a delta-sigma analog-to-digital converter (ADC) is utilized. Accordingly, the measured signals $\mathbf{m}_{k}$ obtained at time instant $k T_{\mathrm{s}}$ are the average values over the previous sampling interval $\left[(k-1) T_{\mathrm{s}}, k T_{\mathrm{s}}\right]$, i.e. [?]

$$
\begin{aligned}
\mathbf{m}_{k} & =\frac{1}{T_{\mathrm{s}}} \int_{(k-1) T_{\mathrm{s}}}^{k T_{\mathrm{s}}} \mathbf{C}_{\mathrm{p}} \mathbf{x}_{\mathrm{p}}(\tau) \mathrm{d} \tau \\
& =\mathbf{H}_{\mathrm{p}} \mathbf{x}_{\mathrm{p}, k-1}+\mathbf{J}_{\mathrm{i}, \mathrm{p}} v_{\mathrm{conv}, k-1}+\mathbf{J}_{\mathrm{o}, \mathrm{p}} v_{\mathrm{PCC}, k-1}
\end{aligned}
$$

where matrix $\mathbf{C}_{\mathrm{p}}$ corresponds to the measured state (i.e. $\mathbf{C}_{\mathrm{p}}^{\mathrm{DM}}=\left[\begin{array}{lll}0 & 1 & 0\end{array}\right]$ and $\mathbf{C}_{\mathrm{p}}^{\mathrm{CM}}=\left[\begin{array}{ll}0 & 1\end{array}\right]$ corresponding to the measured converter current $i_{\text {conv,p }}$ in this work from (1)),

$$
\begin{gathered}
\mathbf{H}_{\mathrm{p}}=\frac{1}{T_{\mathrm{s}}} \int_{(k-1) T_{\mathrm{s}}}^{k T_{\mathrm{s}}} \mathbf{C}_{\mathrm{p}} e^{\tau \mathbf{A}_{\mathrm{p}}} \mathrm{d} \tau \\
\mathbf{J}_{x, \mathrm{p}}=\frac{1}{T_{\mathrm{s}}} \int_{(k-1) T_{\mathrm{s}}}^{k T_{\mathrm{s}}} \int_{(k-1) T_{\mathrm{s}}}^{t} \mathbf{C}_{\mathrm{p}} e^{\tau \mathbf{A}_{\mathrm{p}}} \mathbf{B}_{x, \mathrm{p}} \mathrm{d} \tau \mathrm{d} t, x \in\{\mathrm{i}, \mathrm{o}\} .
\end{gathered}
$$

Since the measurement is inherently one-sample delay, the augmented state-space model is constructed and can be written as

$$
\mathbf{x}_{\text {aug }, k+1}=\mathbf{F}_{\text {aug }} \mathbf{x}_{\text {aug }, k}+\mathbf{G}_{\mathrm{i}, \text { aug }} v_{\text {conv }, k}+\mathbf{G}_{\mathrm{O}, \text { aug }} v_{\mathrm{PCC}, k}
$$

with

$$
\begin{gathered}
\mathbf{x}_{\mathrm{aug}}=\left[\begin{array}{c}
\mathbf{x}_{\mathrm{p}} \\
\mathbf{m}
\end{array}\right], \mathbf{F}_{\mathrm{aug}}=\left[\begin{array}{cc}
\mathbf{F}_{\mathrm{p}} & \mathbf{0} \\
\mathbf{H}_{\mathrm{p}} & \mathbf{0}
\end{array}\right] \\
\mathbf{G}_{\mathrm{i}, \text { aug }}=\left[\begin{array}{c}
\mathbf{G}_{\mathrm{i}, \mathrm{p}} \\
\mathbf{J}_{\mathrm{i}, \mathrm{p}}
\end{array}\right], \mathbf{G}_{\mathrm{o}, \text { aug }}=\left[\begin{array}{c}
\mathbf{G}_{\mathrm{o}, \mathrm{p}} \\
\mathbf{J}_{\mathrm{o}, \mathrm{p}}
\end{array}\right] .
\end{gathered}
$$

With other types of ADCs, the measurement obtained at time instant $k T_{\mathrm{s}}$ can be pointwise and corresponds to the the state information at the same time instant, i.e. $\mathbf{m}_{k}=\mathbf{C}_{\mathrm{p}} \mathbf{x}_{k}$. Consequently, the augmented system in (18) becomes identical to $(10)$.

Herein, the steady-state Kalman filter is used to estimate and predict the state $N$-sample ahead by

$$
\begin{aligned}
\hat{\mathbf{x}}_{\mathrm{aug}, k+N}= & \mathbf{F}_{\mathrm{aug}}^{N} \hat{\mathbf{x}}_{\mathrm{aug}, k}+\sum_{j=0}^{N-1} \mathbf{F}_{\mathrm{aug}}^{N-1-j} \mathbf{G}_{\mathrm{i}, \mathrm{aug}} v_{\mathrm{conv}, k+j} \\
& +\sum_{j=0}^{N-1} \mathbf{F}_{\mathrm{aug}}^{N-1-j} \mathbf{G}_{\mathrm{o}, \text { aug }} \hat{v}_{\mathrm{PCC}, k+j}+\mathbf{F}_{\mathrm{aug}}^{N} \mathbf{K}\left(\mathbf{m}_{k}-\hat{\mathbf{m}}_{k}\right)
\end{aligned}
$$

${ }^{1}$ The anticipative action $v_{\mathrm{vc}, k+N}$ can be easily derived given a desired reference $v_{\mathrm{vc}, k}$. Since the PCC voltage normally is periodic and varies slowly compared to the sampling time, $\hat{v}_{\mathrm{PCC}, k+N}$ can be easily predicted or be approximated as the measured one, i.e. $v_{\mathrm{PCC}, k+N} \approx v_{\mathrm{PCC}, k}$. 


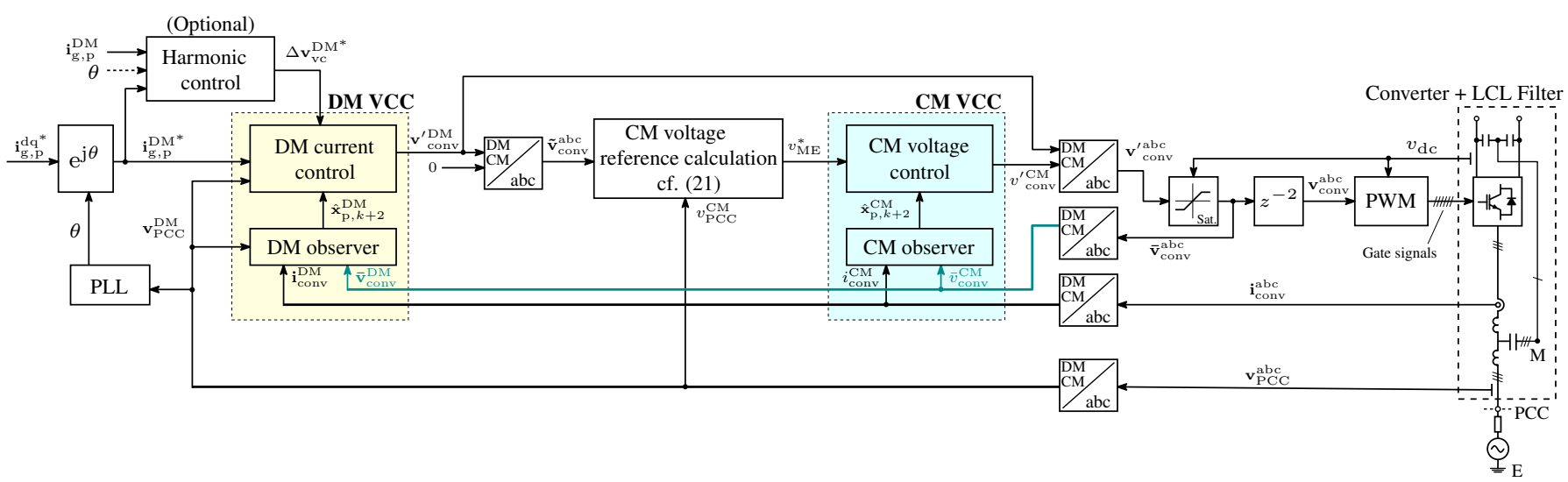

Fig. 5. Simplified VCC system and circuit diagram of the experimental setup and simulation. The transformation between abc and DM/CM herein is the power-invariant Clarke transformation.

where $\mathbf{K}$ is a steady-state Kalman gain [?]. The detailed gain computation is beyond the scope of this work, but can be consulted in [?], [?]. For simplicity, with low latency $(N=2)$ and small sampling time in this work, the measured PCC voltage is used as the estimated one, i.e. $\hat{v}_{\mathrm{PCC}, k+j} \approx v_{\mathrm{PCC}, k}$ [?].

Together, equations (16) and (19) comprise the VCC method.

\section{B. Combining DM and CM VCC Controls}

To extend the modulation index range, the low-frequency component of the DC-bus midpoint voltage w.r.t. the grid earth, $v_{\mathrm{ME}}$ in Fig. 1, should be properly modulated [?]. For the considered three-phase three-wire grid and the power-invariant Clarke transformation, $v_{\mathrm{ME}}$ can be found from

$$
v_{\mathrm{ME}}=\left(v_{\mathrm{PCC}}^{\mathrm{CM}}-v_{\mathrm{C}, \mathrm{p}}^{\mathrm{CM}}\right) / \sqrt{3},
$$

which requires the control of the $\mathrm{CM}$ capacitor voltage $v_{\mathrm{C}, \mathrm{p}}^{\mathrm{CM}}$. The strategy implemented herein is to select the reference $v_{\mathrm{ME}}^{*}$ to center the DM reference voltage with respect to the available DC-bus voltage for carrier-based modulation:

$$
v_{\mathrm{ME}}^{*}=\frac{\max \left\{\tilde{\mathbf{v}}_{\mathrm{conv}}^{\mathrm{abc}}\right\}+\min \left\{\tilde{\mathbf{v}}_{\mathrm{conv}}^{\mathrm{abc}}\right\}}{2}
$$

where $\max \left\{\tilde{\mathbf{v}}_{\text {conv }}^{\text {abc }}\right\}$ and $\min \left\{\tilde{\mathbf{v}}_{\text {conv }}^{\text {abc }}\right\}$ are the maximum and minimum elements in the CM-free vector $\tilde{\mathbf{v}}_{\text {conv }}^{\text {abc }}$ (Fig. 5). From (20), this is equivalent to defining the setpoint

$$
v_{\mathrm{C}, \mathrm{p}}^{\mathrm{CM}^{*}}=v_{\mathrm{PCC}}^{\mathrm{CM}}-\sqrt{3} v_{\mathrm{ME}}^{*} \cdot
$$

Accordingly, $v_{\mathrm{conv}}^{\prime \mathrm{CM}}$ is generated from (16) for controlling $v_{\mathrm{C}, \mathrm{p}}^{\mathrm{CM}}$ to track $v_{\mathrm{C}, \mathrm{p}}^{\mathrm{CM}^{*}}$. Given $\mathbf{v}_{\text {conv }}^{\prime \mathrm{DM}}$ and $v_{\text {conv }}^{\prime \mathrm{CM}}$, the final command voltage $\mathbf{v}_{\text {conv }}^{\text {abc }}$ can be obtained for the converter (Fig. 5).

\section{Auxiliary Control}

Generally, to eliminate the steady-state error, the control system should include the models of system disturbances according to the internal model principle, such as resonant action for zero steady-state error against the sinusoidal grid voltage [?]. We treat this as an auxiliary controller because the resonant damping is relevant to the stability of the system [?].

This auxiliary controller can be divided into two categories: large and narrow bandwidth controllers [?]. The former aims to compensate any deviation instantaneously for fast response such as disturbance observer, while the latter compensates harmonic distortions within a few fundamental periods such as harmonic compensation. These auxiliary controls can be simply integrated into the VCC method.

1) Disturbance observer: The objective is to estimate the equivalent input disturbance $d$, which can be modeled as

$$
\mathbf{d}_{k+1}=\mathbf{F}_{\text {dist }} \mathbf{d}_{k} .
$$

For example, to recursively track a slowly time-varying unknown signal, an observer should possess a fading memory by weighing the recent information more strongly than the old one to maintain stability against stochastic noise after convergence [?]. Therefore, the integral action by state $d_{\mathrm{I}}$ is included with a forgetting factor, resulting in the disturbance model where $\mathrm{e}^{\alpha T_{\mathrm{s}}}$ with $\alpha<0$, and

$$
\mathbf{d}=\left[\begin{array}{c}
d \\
d_{\mathrm{I}}
\end{array}\right], \mathbf{F}_{\text {dist }}=\left[\begin{array}{cc}
\mathrm{e}^{\alpha T_{\mathrm{s}}} & 0 \\
1 & 1
\end{array}\right] .
$$

The model of $\mathbf{F}_{\text {dist }}$ for a sinusoidal disturbance, such as grid harmonics, or resonant actions can be found in [?], [?].

Subsequently, (23) can be integrated with (18) to design the new observer by using the new augmented state $\tilde{\mathbf{x}}_{\text {aug }}=$

$$
\begin{gathered}
{\left[\begin{array}{cc}
\mathbf{x}_{\text {aug }} & \mathbf{d}
\end{array}\right]^{T} \text { and replacing } \mathbf{F}_{\text {aug }}, \mathbf{G}_{\mathrm{i}, \text { aug }} \text { and } \mathbf{G}_{\mathrm{o}, \text { aug }} \text { with }} \\
\widetilde{\mathbf{F}}_{\text {aug }}=\left[\begin{array}{cc}
\mathbf{F}_{\text {aug }} & \mathbf{G}_{\text {aug,dist }} \\
\mathbf{0} & \mathbf{F}_{\text {dist }}
\end{array}\right], \mathbf{G}_{\text {aug }, \text { dist }}=\left[\begin{array}{ll}
\mathbf{G}_{\mathrm{i}, \text { aug }} & \mathbf{0}
\end{array}\right] \\
\widetilde{\mathbf{G}}_{\mathrm{i}, \text { aug }}=\left[\begin{array}{c}
\mathbf{G}_{\mathrm{i}, \text { aug }} \\
\mathbf{0}
\end{array}\right], \widetilde{\mathbf{G}}_{\mathrm{o}, \text { aug }}=\left[\begin{array}{c}
\widetilde{\mathbf{G}}_{\mathrm{o}, \text { aug }} \\
\mathbf{0}
\end{array}\right] .
\end{gathered}
$$

To nullify the effect of the disturbance, the control law in (16) is updated by feedforwarding the estimated disturbance as

$$
v_{\text {conv, new }}^{\prime}=v_{\text {conv,old }}^{\prime}+\hat{d} .
$$

2) Harmonic control or compensation: To mitigate the grid-current distortion, e.g. due to low-order harmonics of 

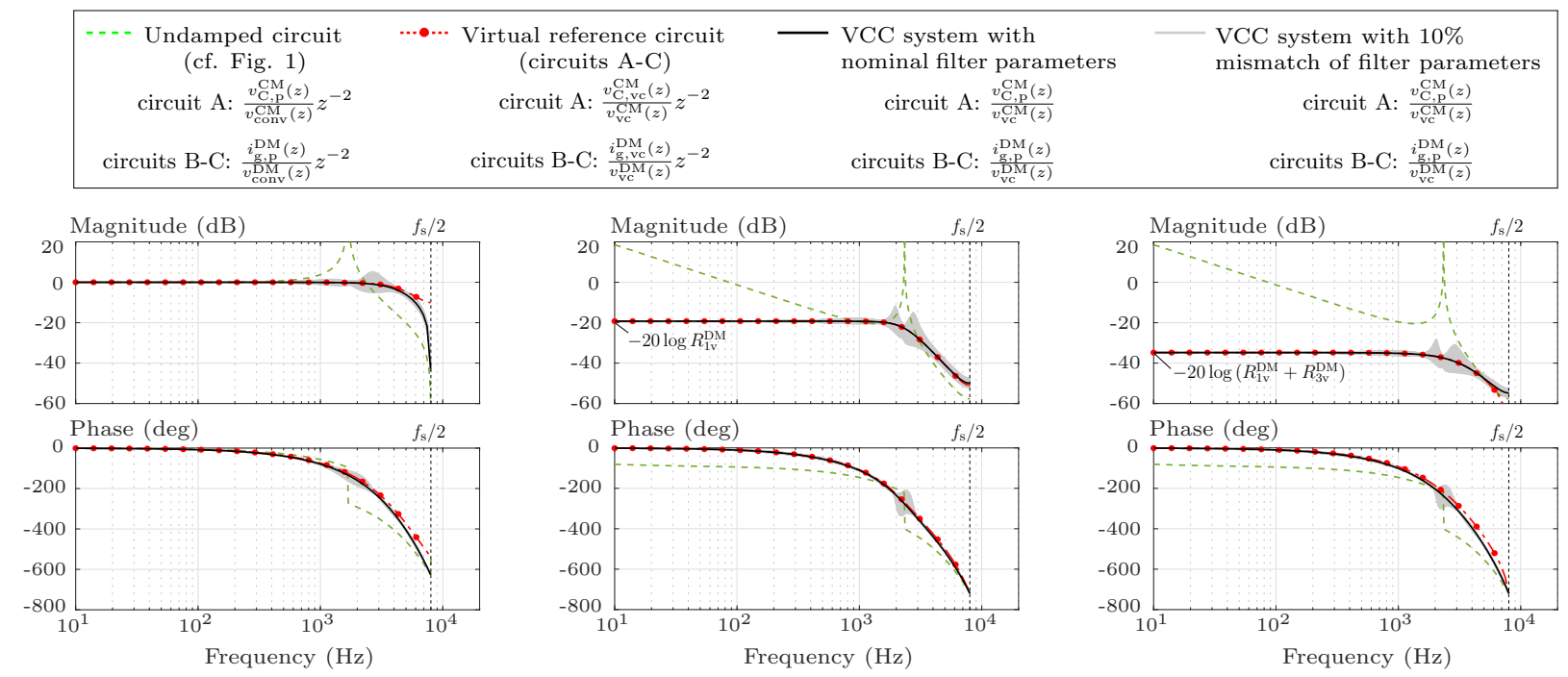

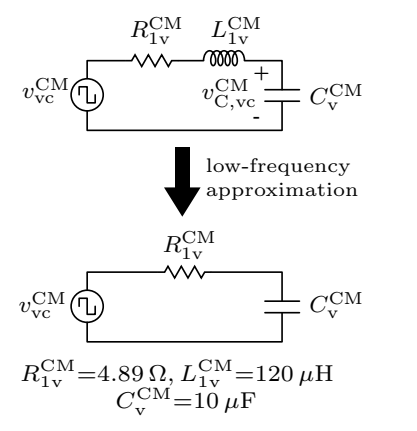

(a) Circuit A

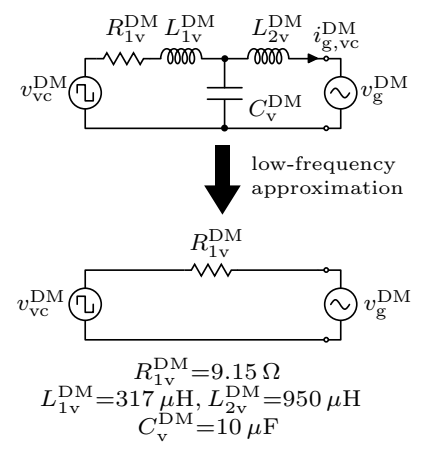

(b) Circuit B

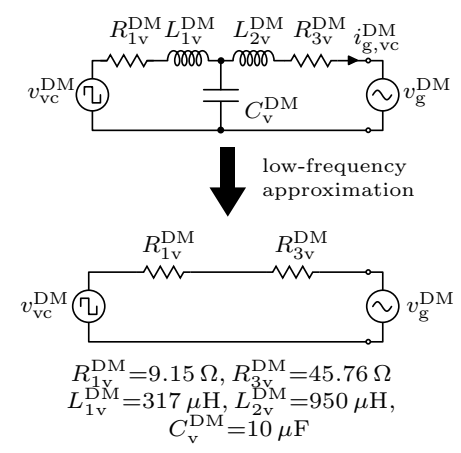

(c) Circuit C

Fig. 6. Examples of virtual circuit designs for the converter with the lossless $L C L$ filter. (Top) Frequency responses of input-to-output transfer functions with $z=e^{\mathrm{j} \omega T_{\mathrm{s}}}$. For fair comparison, the effect of 2-sample computational delay is also included for the responses of the physical circuit and virtual circuit. The $10 \%$ tolerance of filter components is considered through Monte-Carlo simulation (grey lines). (Middle) Virtual reference circuits for the VCC method. (Bottom) Low-frequency approximations of the virtual circuits for designing current and voltage controllers.

the grid voltage and the deadtime, a harmonic control or compensation (including the fundamental frequency) is indispensable [?], [?], e.g. using resonant control, repetitive control, and discrete Fourier transform (DFT)-based selective harmonic compensation (see details in [?]). This can be plugged in with the VCC method as shown in Fig. 5.

\section{Virtual Reference Circuit Design Examples}

The design of the virtual reference circuit is crucial to the VCC method. Through this circuit, designers can specify the desired properties of the closed-loop controlled converter. In particular, existing theories of filter network can be of use.

As mentioned in Section II, the parameters of the virtual circuit in Fig. 2, nevertheless, should not be selected arbitrarily. The physical circuit and the virtual circuit should resemble each other sufficiently well. This can be roughly indicated by the condition number of the transformation matrix $\mathbf{T}_{\mathrm{d}}$. As suggested by (8) and Fig. $4, L_{1 \mathrm{v}}$ and $R_{1 \mathrm{v}}$ in DM/CM virtual circuits can be arbitrarily set without affecting the transformation when the circuit bandwidth is sufficiently lower than the Nyquist frequency. In other words, a good virtual circuit should be similar to the physical circuit and only some elements next to the converter are allowed to be added or adjusted, e.g. $L_{2 \mathrm{v}}^{\mathrm{DM}}=L_{2 \mathrm{p}}^{\mathrm{DM}}$ and $C_{\mathrm{v}}^{\mathrm{CM}}=C_{\mathrm{p}}^{\mathrm{CM}}$. This suggests a component-sizing and control co-design method during the early filter-design process [?]. Still, adjusting the other elements is possible as illustrated in this section.

As notable examples, three virtual circuits, namely A, B and $\mathrm{C}$, are considered for designing the AD-based controllers using the VCC method (Fig. 6). Circuit A is considered for the CM case, whereas circuits $\mathrm{B}$ and $\mathrm{C}$ are considered for the $\mathrm{DM}$ case. Circuits A and B are designed using the CM and DM circuits, respectively, with a single series resistor at the converter side (Fig. 3). Therefore, for $\mathrm{CM}$ virtual circuit, $R_{1 \mathrm{v}}^{\mathrm{CM}}$ is necessary since using only $R_{2 \mathrm{v}}^{\mathrm{CM}}$ in parallel to the capacitor will lead to excessive current overshoot. As previously discussed, these virtual circuits are selected to differ from the physical circuit only in the part next to the converter. Nevertheless, circuit $\mathrm{C}$ includes the series resistor $R_{3 \mathrm{v}}^{\mathrm{DM}}$ at the grid side and proves useful for counteracting grid-impedance variations. Note that a circuit with the resistor $R_{2 \mathrm{v}}^{\mathrm{DM}}$ in parallel with the capacitor commonly found in the literature [?], [?] can also be used [?].

The frequency responses of the VCC system based on circuits A-C and the filter parameters in Table I are shown in Fig. 6 (top). Since the VCC system is observer based, the effect of filter component variations is considered. The Monte Carlo 
TABLE I

SYSTEM PARAMETERS

\begin{tabular}{lcc}
\hline \multicolumn{3}{c}{ Nominal values } \\
\hline Rated power & $P_{\mathrm{n}}$ & $15 \mathrm{~kW}$ \\
Grid voltage & $V_{\mathrm{g}}$ & $230 \mathrm{~V}_{\mathrm{rms}}$ \\
Rated current & $I_{\mathrm{n}}$ & $21 \mathrm{~A}_{\mathrm{rms}}$ \\
Grid frequency & $f_{\mathrm{g}}$ & $50 \mathrm{~Hz}$ \\
\hline & LCL filter & \\
\hline Converter-side inductance & $L_{1 \mathrm{p}}$ & $0.9 \mathrm{mH}(0.027 \mathrm{pu})$ \\
Grid-side inductance & $L_{2 \mathrm{p}}$ & $0.95 \mathrm{mH}(0.028 \mathrm{pu})$ \\
Filter capacitance & $C_{\mathrm{p}}$ & $10 \mu \mathrm{F}(10.58 \mathrm{pu})$ \\
DM filter resonance & $f_{\text {res }}^{\mathrm{DM}}$ & $2.34 \mathrm{kHz}$ \\
CM filter resonance & $f_{\text {res }}^{\mathrm{CM}}$ & $1.68 \mathrm{kHz}$ \\
\hline
\end{tabular}

Experiment with grid impedance (Section VI-B)

\begin{tabular}{lcc}
\hline $\begin{array}{l}\text { Grid inductance } \\
\text { Grid resistance }\end{array}$ & $\begin{array}{c}L_{\mathrm{g}} \\
R_{\mathrm{g}}\end{array}$ & $\begin{array}{c}1.63 \mathrm{mH}(0.048 \mathrm{pu}) \\
2 \Omega(0.19 \mathrm{pu})\end{array}$ \\
\hline \multicolumn{3}{c}{ Converter and controller } \\
\hline Switching frequency & $f_{\mathrm{sw}}$ & $16 \mathrm{kHz}$ \\
Dead time & $t_{\mathrm{dead}}$ & $2 \mu \mathrm{s}$ \\
DC-bus voltage (DC supply) & $v_{\mathrm{dc}}$ & $700 \mathrm{~V}$ \\
Sampling frequency (single update) & $f_{\mathrm{s}}$ & $16 \mathrm{kHz}$ \\
\hline
\end{tabular}

simulation was conducted based on 200 sets of component values $\left\{L_{1 \mathrm{p}}, L_{2 \mathrm{p}}, C_{\mathrm{p}}\right\}$ in Fig. 1, which were independently generated from the uniform distribution with the interval of $\pm 10 \%$ deviation from the nominal values in Table I. For each set, the frequency response of the VCC system was computed and plotted.

A. CM and DM Virtual Circuits with Series Resistors $R_{1 \mathrm{v}}^{\mathrm{CM}}$ and $R_{1 \mathrm{v}}^{\mathrm{DM}}$ (Circuits $A$ and $B$ )

This circuit topology coincides with a singly-terminated ladder network [?], which can also be extended to the sizing of filter components [?]. In this example, given a desired bandwidth $\omega_{\mathrm{bw}}=2 \pi f_{\mathrm{bw}}$, the virtual circuits are designed based on the well-known Butterworth circuits in Fig. 3. Equivalently, the closed-loop poles are placed at the same distance from the origin in CT domain yielding an efficient control action [?].

1) Parameter selection: Firstly, $L_{2 \mathrm{v}}^{\mathrm{DM}}=L_{2 \mathrm{p}}^{\mathrm{DM}}$ and $C_{\mathrm{v}}^{\mathrm{CM}}=C_{\mathrm{p}}^{\mathrm{CM}}$ are selected for circuits $\mathrm{B}$ and $\mathrm{A}$, respectively. For DM VCC, circuit B is considered with $f_{\mathrm{bw}}^{\mathrm{DM}}=2.34 \mathrm{kHz}$, which is the same as the DM filter resonant frequency ( $f$ res in Table I). In general, if the control bandwidth is significantly lower than the resonant frequency in a physical circuit, it can have a major impact on the system based on the sampling frequency selection and introduce unacceptable sensitivities to unmodeled disturbances [?]. On the other hand, pushing the bandwidth higher than the resonant frequency can lead to an aggressive control action when fast signals are commanded because of high attenuation of $L C L$ filters above the resonant frequency [?].

Subsequently, for CM VCC, circuit A is considered with $f_{\mathrm{bw}}^{\mathrm{CM}}=5 \mathrm{kHz}$, which is higher than twice the DM bandwidth of circuit $\mathrm{B}\left(f_{\mathrm{bw}}^{\mathrm{DM}}\right)$. This aims for the fast reference tracking of $v_{\mathrm{C}, \mathrm{p}}^{\mathrm{CM}}$ and $v_{\mathrm{ME}}$. According to Fig. 3, the component values of the designed virtual circuits A and B are shown in Figs. $6 a \& b$.

Alternatively, the bandwidth can be chosen based on the dominant pole selection and the desired slew rate [?], [?].

2) VCC system frequency response and low-frequency behavior: According to Figs. 6a\&b (top), the CM and DM resonances are properly damped with circuits A and B respectively. The result of VCC with circuit B based on nominal filter parameters matches the desired response of circuit B. For circuit A, the bandwidth is high relative to the Nyquist frequency $\left(f_{\mathrm{s}} / 2=8 \mathrm{kHz}\right)$ and higher than the $\mathrm{CM}$ resonant frequency, causing some discrepancies at high frequency near the Nyquist frequency (see also Fig. 4c).

From the circuit viewpoint, at low frequency, circuits A and B approximate a series $R C$ circuit and a circuit with series resistor $R_{1 \mathrm{v}}^{\mathrm{DM}}$ (Figs. 6a\&b, bottom). This is also confirmed by the corresponding flat magnitude response with phase around $0^{\circ}$ at low frequency (Figs. 6a\&b, top).

Under filter parameter variations, the filter resonant frequency slightly shifts and causes a new resonance around the nominal resonant frequency $(2.34 \mathrm{kHz})$. Nevertheless, the VCC system remains stable; the new resonances are damped, showing the small magnitude (Figs. 6a\&b, top).

3) Voltage and current control laws: Another benefit of these virtual circuits is the simplified resistive behavior at low frequency, facilitating the design of the current and voltage controller. According to circuit A at low frequency (Fig. 6a, bottom), given the setpoint $v_{\mathrm{C}}^{\mathrm{CM}^{*}}$ in (22), the control law for $v_{\mathrm{vc}}^{\mathrm{CM}}$ can simply be

$$
v_{\mathrm{vc}, k}^{\mathrm{CM}}=v_{\mathrm{C}, \mathrm{p}, k}^{\mathrm{CM}^{*}} .
$$

According to circuit B at low frequency (Fig. 6b, bottom), to inject the grid current according to the setpoint $i i_{\mathrm{g}, \mathrm{p}}{ }^{*}$ into the grid voltage $v_{\mathrm{g}}^{\mathrm{DM}}$, the control law for $v_{\mathrm{vc}}^{\mathrm{DM}}$ becomes a simple feedforward law as

$$
v_{\mathrm{vc}, k}^{\mathrm{DM}}=R_{1 \mathrm{v}}^{\mathrm{DM}} i_{\mathrm{g}, \mathrm{p}, k}^{\mathrm{DM}^{*}}+v_{\mathrm{g}, k}^{\mathrm{DM}}+\Delta v_{\mathrm{vc}, k}^{\mathrm{DM}}
$$

where $\Delta v_{\mathrm{vc}, k}^{\mathrm{DM}}$ can be added by supervisory loops (Fig. 5) such as a resonant controller [?] and DFT-Based selective harmonic compensation for harmonic control (Section VI-C2).

\section{B. DM Virtual Circuit with Two Series Resistors $R_{1 \mathrm{v}}^{\mathrm{DM}}$ and $R_{3 \mathrm{v}}^{\mathrm{DM}}$ (Circuit $C$ )}

Ensuring the stability of VSCs connected to a grid with unknown and large grid impedance is challenging, particularly for VSCs with an observer-based control. Although stabilizing the system can be done through adjusting a controller or an observer, this example focuses on adjusting a controller through the VCC method. Under unknown grid impedance variations, for a given observer, a controller which can stabilize a system proves more robust.

Intuitively, a grid impedance might resonate with a filter capacitor resulting in a resonant current, which the single converter-side resistor $R_{1 \mathrm{v}}^{\mathrm{DM}}$ cannot sufficiently damp. From the VCC viewpoint, to strengthen the damping of the grid 


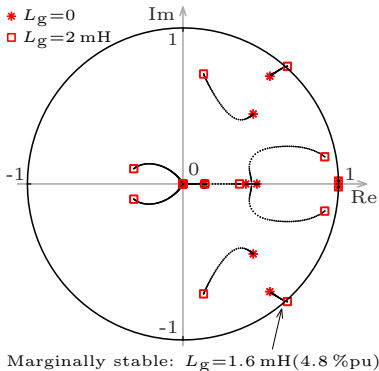

(a) Circuit $\mathrm{B}$

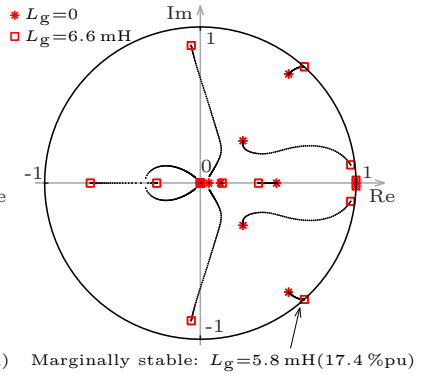

(b) Circuit $\mathrm{C}$

Fig. 7. Eigenvalue loci of the complete DT VCC system under grid inductance variations using DM virtual circuits $\mathrm{B}$ and $\mathrm{D}$. The grid inductance varies (a) from 0 to $2 \mathrm{mH}$ and (b) from 0 to $6.6 \mathrm{mH}$. Note that this grid inductance is not considered in the VCC design.

current, an additional grid-side resistance $R_{3 \mathrm{v}}^{\mathrm{DM}}$ is introduced (Fig. 6c). Thanks to the VCC method, the physical grid current $i_{\mathrm{g}, \mathrm{p}}$ tends to inherit the property of the virtual circuit counterpart $i_{\mathrm{g}, \mathrm{vc}}$.

As a compromise between the damping effectiveness and the robustness of the model-following control when including $R_{3 \mathrm{v}}^{\mathrm{DM}}$ (cf. Fig. $4 \mathrm{~b}$ ), circuit $\mathrm{C}$ is designed from selecting $R_{3 \mathrm{v}}^{\mathrm{DM}}=5 R_{1 \mathrm{v}}^{\mathrm{DM}}$ and added to circuit $\mathrm{B}$. Considering the eigenvalue loci of the VCC system under grid inductance variations ${ }^{2}$ in Fig. 7, using circuit B (Fig. 6b) results in the critical grid inductance that destabilizes the system of $L_{\mathrm{g}}=1.6 \mathrm{mH}\left(1.68 L_{2 \mathrm{p}}\right)$. However, using circuit $\mathrm{C}$ with the chosen $R_{3 \mathrm{v}}^{\mathrm{DM}}$ (Fig. 6c) results in the higher critical grid inductance of $L_{\mathrm{g}}=5.8 \mathrm{mH}\left(6.1 L_{2 \mathrm{p}}\right)$. From our observation, a greater increase in $R_{3 \mathrm{v}}^{\mathrm{DM}}$ can further improve the system robustness against grid inductance variations. Equivalently, knowing $L_{\mathrm{g}}$, the system eigenvalue loci regarding $R_{3 \mathrm{v}}^{\mathrm{DM}}$ can also be computed. In other words, circuit $\mathrm{C}$ generates a set of eigenvalues that are more robust, and are different from the set of poles generated by circuit B (cf. Fig. 7a\&b at $L_{\mathrm{g}}=0$ ). Note that $R_{1 \mathrm{v}}^{\mathrm{DM}}$ is always required in circuit $\mathrm{C}$ for the effective damping of the nominal $L C L$ filter. Implementing only large $R_{3 \mathrm{v}}^{\mathrm{DM}}$ might lead to less robustness of the model-following control and thus ineffective damping even under a normal grid compared to circuit B.

Considering the frequency response, the similar response to circuit B can both be observed with nominal filter parameters and under component variations (Fig. 6c, top). One difference is that slightly more deviations can be observed at high frequency despite nominal filter parameters. This is because circuit $\mathrm{C}$ (with $R_{3 \mathrm{v}}^{\mathrm{DM}}$ ) is less compatible with the physical circuit than circuit B (cf. Fig. 4a\&b).

Similar to circuit B, at low frequency, circuit C approximates a circuit with series resistor $R_{1 \mathrm{v}}^{\mathrm{DM}}+R_{3 \mathrm{v}}^{\mathrm{DM}}$ (Fig. 6c, bottom). Therefore, the control law for $v_{\mathrm{vc}}^{\mathrm{DM}}$ is the same as (27) but with $R_{1 \mathrm{v}}^{\mathrm{DM}}+R_{3 \mathrm{v}}^{\mathrm{DM}}$ instead of $R_{1 \mathrm{v}}^{\mathrm{DM}}$.

\section{Simulation AND ExPerimental Results}

To verify the performance of the VCC method based on the designed virtual circuits in Fig.6, both the simulations

\footnotetext{
${ }^{2}$ Since a grid resistance can improve a damping of a system, only a pure inductance $L_{\mathrm{g}}$ is considered as a grid impedance to draw the worst case [?].
}

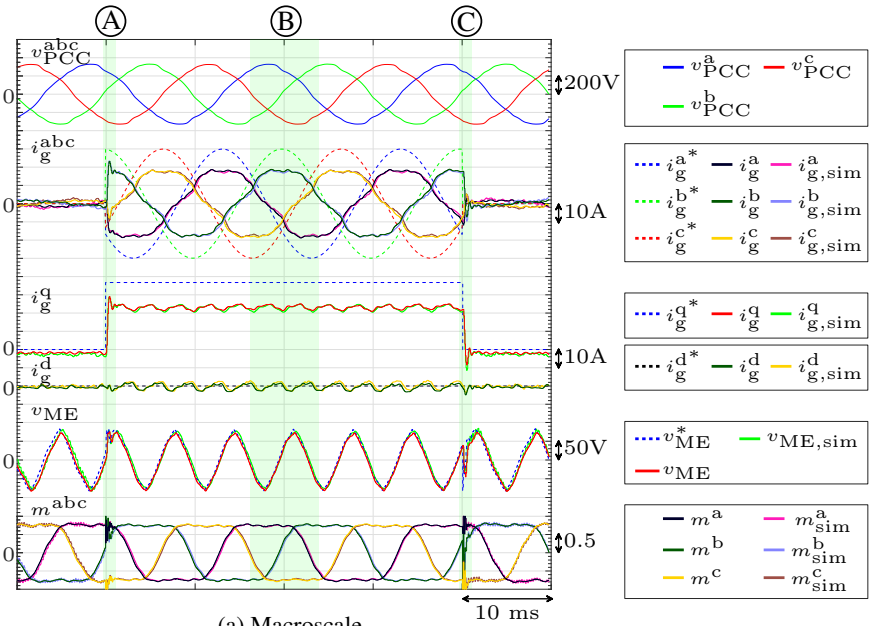

(a) Macroscale

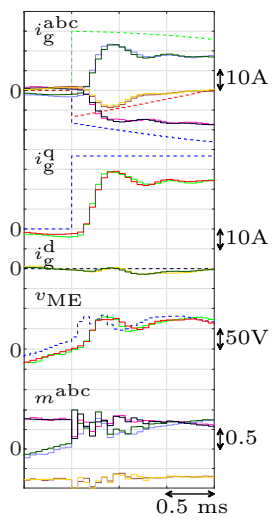

(b) Zoom A

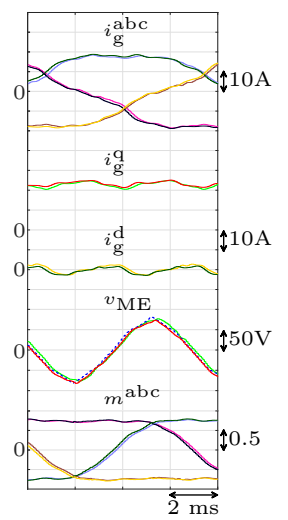

(c) Zoom B

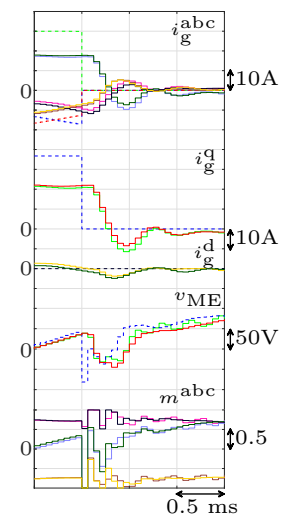

(d) Zoom C
Fig. 8. Experimental (without subscript) and simulation (subscript 'sim') results for a reference step up and down of grid reactive current $i_{\mathrm{g}, \mathrm{p}}^{\mathrm{q}}$ using virtual circuits $\mathrm{B}$ and $\mathrm{A}$ for damping DM and $\mathrm{CM}$ resonances. The three zooms (down) demonstrate the response during transient and steady state.

and the experiments were conducted based on the VSC with $L C L$ filter in Fig. 1a and the system parameters in Table I. The simplified diagram of the VCC system and experimental setup are displayed in Fig. 5. Unless otherwise stated, only the converter currents $i_{\text {conv,p }}$ and the PCC voltages $v_{\mathrm{PCC}}$ are measured and employed for the VCC method. This corresponds to the case where only the current sensor available is of the converter current for the purpose of converter protection. The other measurements were only used for result illustrations.

The DC bus voltage was supplied by a regulated DC power supply. To avoid overloading the supply, only step changes of the grid reactive current were applied to excite any potential resonances. The VCC controller was experimentally implemented in the Triphase 3PExpress rapid-prototype platform (Table I) [?]. The platform provides real-time control and monitoring with 2-sample computational delay (latency $N=2)^{3}$ and integrated measurement system based on delta-sigma ADCs.

The simulation was performed in MATLAB based on a linear average model of VSCs including the average model of

\footnotetext{
${ }^{3}$ In most digitally controlled power converters, the computational delay is one sample or $N=1$.
} 


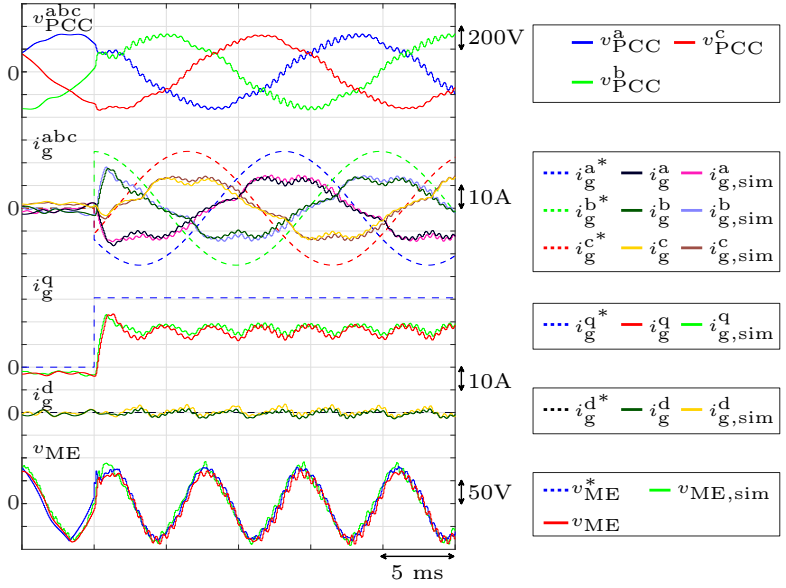

(a) circuit B (with $R_{1 \mathrm{v}}^{\mathrm{DM}}$ )

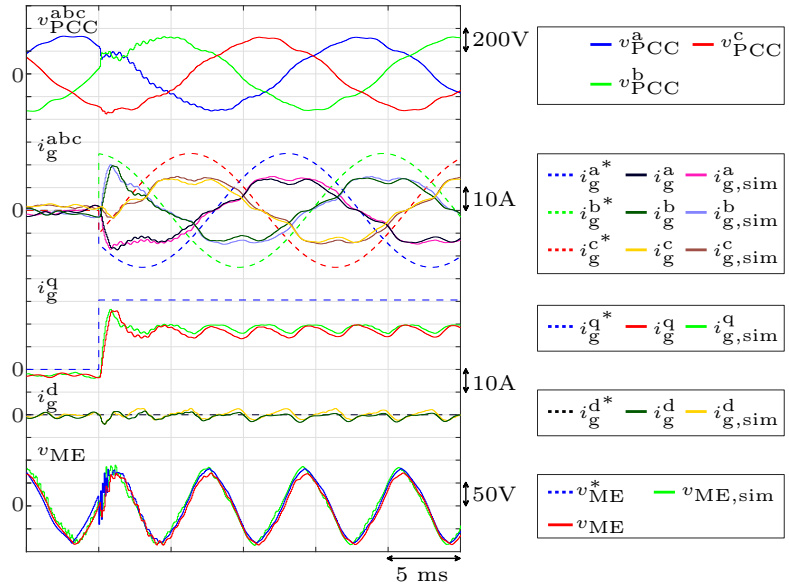

(b) circuit C (with $R_{1 \mathrm{v}}^{\mathrm{DM}}$ and $R_{3 \mathrm{v}}^{\mathrm{DM}}$ )

Fig. 9. Experimental (without subscript) and simulation (subscript 'sim') results for a reference step up grid reactive current $i_{\mathrm{g}, \mathrm{p}}^{\mathrm{q}^{*}}$ using virtual (a) circuit B and (b) circuit $\mathrm{C}$ under grid inductance of $L_{\mathrm{g}}=1.63 \mathrm{mH}$ (Table I). As predicted in Fig. 7a, the VCC system with circuit B becomes marginally stable (a), while the one with circuit $\mathrm{C}$ is stable (b).

deadtime [?], [?]. The harmonics present in the experimental grid voltage were characterized and used in the simulation. Hereinafter, the asterisk $*$ denotes the reference waveforms.

To focus on verifying damping performance and stability, without loss of generality [?], no harmonic control or resonant action is implemented $\left(\Delta v_{\mathrm{vc}}^{\mathrm{DM}}=0\right.$ in (27)) in the first two subsections (VI-A and VI-B). Then, the last subsection demonstrates the use of disturbance observer and harmonic compensation by adding $\Delta v_{\mathrm{vc}}^{\mathrm{DM}}$ as mentioned in Section IV-C.

\section{A. Grid without Additional Grid Impedance}

A step up and down of grid reactive current $i_{\mathrm{g}, \mathrm{p}}^{\mathrm{q}^{*}}$ in $d q$ frame from $0 \mathrm{~A}$ to the rating current $36.74 \mathrm{~A}$ (amplitude of $30 \mathrm{~A}$ in $a b c$ frame), with $i_{\mathrm{g}, \mathrm{p}}^{\mathrm{d}^{*}}=0$, is applied to observe the dynamic performance of the controllers.

To control the grid current, the input voltages $v_{\mathrm{vc}}^{\mathrm{DM}}$ for virtual circuits $\mathrm{B}$ and $\mathrm{C}$ are calculated in the stationary reference frame from (27). To control the DC-bus midpoint voltage w.r.t. the grid earth $v_{\mathrm{ME}}$, the input voltage $v_{\mathrm{vc}}^{\mathrm{CM}}$ for circuit A is computed from (22) and (26). The experimental results are superimposed on the simulation results and displayed in Fig. 8.

According to Fig. 8, the controller based on circuits B and A properly damps the DM resonance in the grid current $i_{\mathrm{g}, \mathrm{p}}$ and the $\mathrm{CM}$ resonance in the DC-bus midpoint voltage $v_{\mathrm{ME}}$, respectively. In $d q$ frame, the reactive grid current $i_{\mathrm{g}, \mathrm{p}}^{\mathrm{q}}$ shows a mild overshoot after the step up and down as a characteristic of the Butterworth circuit [?]. The grid current suffers from harmonic distortion and about $33 \%$ steady-state error in amplitude mainly because of the grid harmonics and the converter deadtime [?]. None of the models of these disturbances have been considered in the VCC design (Fig. 6b, top), resulting in nonzero steady-state error based on the internal model principle [?].

During the transient, $v_{\mathrm{ME}}$ is also affected by the current step change since $v_{\mathrm{ME}}^{*}$ is derived from the DM step reference $v_{\mathrm{vc}}^{\mathrm{DM}}$ based on (21). At steady state, $v_{\mathrm{ME}}$ becomes a triangular waveform corresponding to the space-vector centering. The modulation function $m=\frac{v_{\text {conv }}}{v_{\text {dc }} / 2}$ is also displayed.

The simulated responses are in excellent agreement with the experimental ones in all the tests despite using the simple linear average model with deadtime. Some small discrepancies stem from unmodeled dynamics e.g. nonlinear nature of the VSC switching and filter components. Notably, the interaction between the controller and the phase-locked loop (PLL) are negligible from the observations due to the low PLL bandwidth [?].

\section{B. Grid with Additional Grid Impedance}

To experimentally verify the performance of the controller based on circuit $\mathrm{C}$ against grid impedance variations, a grid inductance $L_{\mathrm{g}}=1.6 \mathrm{mH}$ is added (Table I). From Fig. 7a, it is the minimum grid inductance that destabilizes the VCC system using the controller based on circuit B. Note that this added inductance is unknown to the controller.

Using circuit $\mathrm{B}$, the grid current $i_{\mathrm{g}, \mathrm{p}}$ and the PCC voltage $v_{\text {PCC }}$ oscillate at high frequency after a reactive current step up (Fig. 9a). This exhibits marginal stability as indicated in Fig. 7a. In both simulation and experiment, the observed natural frequencies of the oscillation are comparably $2.15 \mathrm{kHz}$, which matches the calculated value from Fig. 7a. Although the CM system stability ideally is not affected by the grid impedance, the voltage reference $v_{\mathrm{vc}}^{\mathrm{CM}^{*}}$ is derived in (21) and (26) from the oscillating DM system, resulting in the oscillation of $v_{\mathrm{ME}}^{*}$ and thus $v_{\mathrm{ME}}$.

However, the VCC system with circuit $\mathrm{C}$ is stable after the same step up (Fig. 9b). Although some oscillations can be seen during the step up, they vanish after $5 \mathrm{~ms}$, yielding well-damped responses in both $i_{\mathrm{g}, \mathrm{p}}$ and $v_{\mathrm{ME}}$.

Using the direct pole-placement technique, it is difficult to intuitively adjust the desired closed-loop pole locations to counteract unknown grid impedance. In other words, the eigenvalue loci can be significantly different based on the chosen different closed-loop pole locations (cf. Figs. 7 a\&b). 
(A)

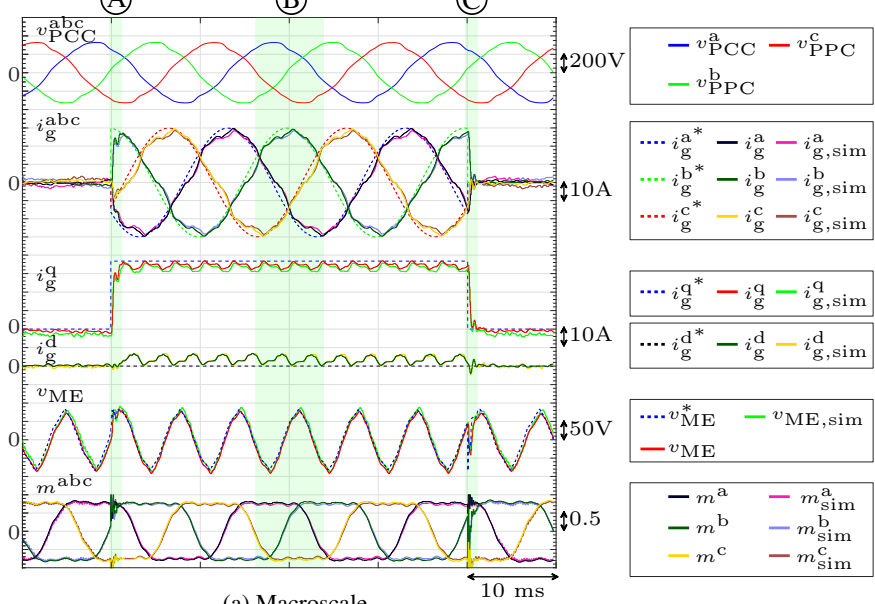

(a) Macroscale

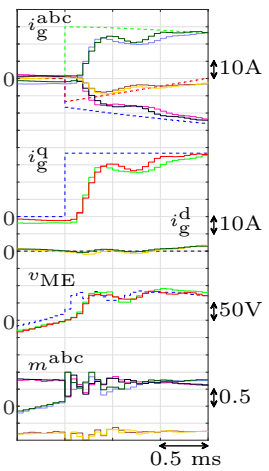

(b) Zoom A

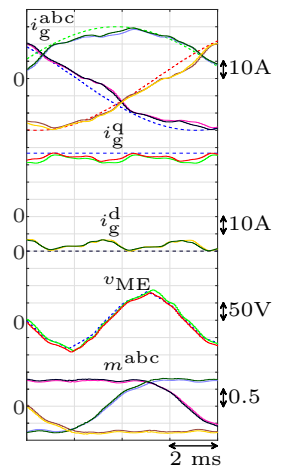

(c) Zoom B

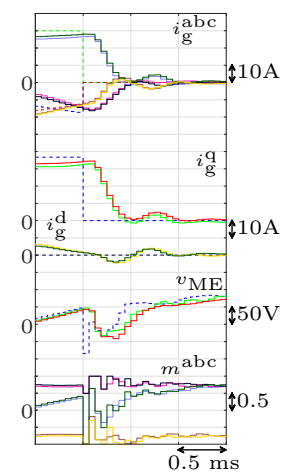

(d) Zoom C
Fig. 10. Experimental (without subscript) and simulation (subscript 'sim') results for a reference step up and down of grid reactive current $i_{\mathrm{g}, \mathrm{p}}^{\mathrm{q}^{*}}$ using the disturbance observer to improve the steady-state response in Fig. 8. The three zooms (down) demonstrate the response during transient and steady state.

However, the VCC method suggests an intuitive way to adjust a controller against a weak grid. The main advantage is the stabilization of the system. Subsequently, an auxiliary controller can be plugged in to improve the distortion (see Section VI-C2) or to on-line identify the grid impedance for achieving long-term robust performance [?], [?].

\section{Integration with Auxiliary Control}

According to Figs. 8 and 9, the control in (27) with $\Delta v_{\mathrm{vc}}^{\mathrm{DM}}=0$ seems insufficient to mitigate the harmonic distortion and the steady-state error in the grid current due to unmodeled disturbance dynamics. To overcome this, as described in Section IV-C, the auxiliary controllers can be seamlessly added with VCC method.

1) Disturbance observer: The disturbance observer in (23) and (24) with $\alpha=-0.1$ is implemented together with the VCC controller based on circuit B. Accordingly, the amplitude error at steady state of $i_{\mathrm{g}, \mathrm{p}}$ vanishes (Fig. 10). Still, the grid current is distorted without a resonant action and harmonic control ${ }^{4}$ [?]. It also slightly lags the setpoint $i_{\mathrm{g}, \mathrm{p}}^{*}$ due to the inherent

\footnotetext{
${ }^{4}$ The detailed implementation of resonant actions with a disturbance observer can be found in [?], [?].
}

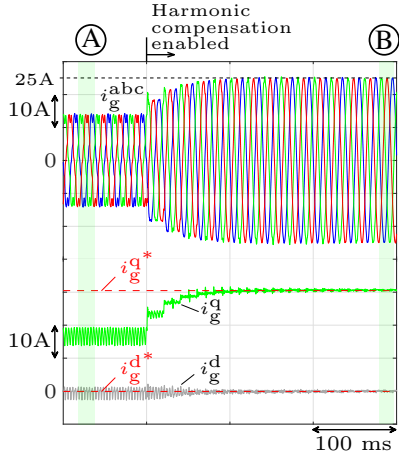

(a) $i_{\mathrm{g}}^{\mathrm{abc}}$ and $i_{\mathrm{g}}^{\mathrm{dq}}$ (Macroscale)
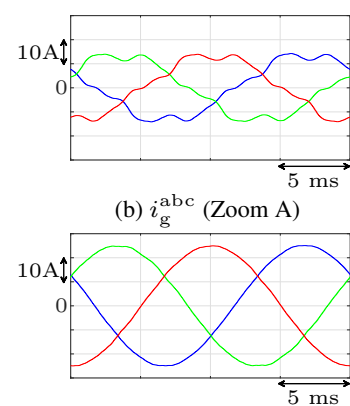

(c) $i_{\mathrm{g}}^{\mathrm{abc}}($ Zoom B)
Fig. 11. Experimental results using virtual circuit $\mathrm{C}$ and DFT-based selective harmonic compensation [?] to improve the steady-state response in Fig. 9 under grid impedance. The two zooms (right) demonstrate the response before and after the compensation.

phase shift of the underlying virtual circuit B (see the phase response in Fig. 6b, top). This causes the slight increase of the active current $i_{\mathrm{g}, \mathrm{p}}^{\mathrm{d}}$.

The convergence of the disturbance observer can be clearly seen during the step-up transient of $i_{\mathrm{g}, \mathrm{p}}^{\mathrm{q}}$ with the settling time around $1.5 \mathrm{~ms}$ and no overshoot (Fig. 10). Before the convergence, the response is similar to the result without a disturbance observer (Fig. 8), showing comparable rise time.

2) Harmonic compensation: The DFT-based selective harmonic compensation in [?] is implemented through $\Delta v_{\mathrm{vc}}^{\mathrm{DM}}$ in (27) (cf. Fig. 5) to enhance the performance of the controller based on circuit $\mathrm{C}$ under the additional grid impedance in Fig.9b. Similar to a bank of parallel resonant controllers, this is an effective implementation of the simultaneous compensation at multiple frequencies. For conciseness, the harmonic compensation also includes the fundamental line-frequency component of $50 \mathrm{~Hz}$. For convenience $^{5}$, the grid current is fed back with lower sampling frequency of $f_{\mathrm{s}} / 2$.

The harmonic compensation is enabled after the system reaches steady state (Fig. 11). Before compensation, the grid current $i_{\mathrm{g}, \mathrm{p}}$ suffers from distortion and amplitude errors (Fig. 11b). However, after enabling the compensation, $i_{\mathrm{g}, \mathrm{p}}$ converges to the setpoint within 5 fundamental periods $(0.1 \mathrm{~s})$, and the waveform quality is significantly improved (Fig. 11c).

\section{CONCLUSIONS}

The VCC method has been proposed to design an AD-based controller for grid-tied VSCs with DM $(L C L)$ and CM $(L C)$ output filters (with respect to current- and voltage-controlled converters). A virtual circuit is an alternative representation of the desired closed-loop poles, offering an intuitive representation for electrical engineers. In other words, the controller design boils down to the design of a virtual circuit. As a result, the physical DM grid current and CM capacitor voltage inherit the properties of the virtual counterparts, e.g. resonance damping and low-frequency behavior. On this

\footnotetext{
${ }^{5}$ The harmonic compensation of the grid currents without grid-current feedback is feasible, but at the expense of the degraded performance [?].
} 
account, the existing filter network theories can be exploited to design the virtual circuit.

Three notables virtual circuits (circuits A-C) have been considered to damp the DM and CM resonances. Circuits $\mathrm{A}$ and $\mathrm{B}$ are based on the well-established Butterworth filters with single series resistor at the converter side. To strengthen the controller against grid impedance variations, a series resistor is added at the grid side, yielding circuit $\mathrm{C}$. Considering the design of the current control, according to the low-frequency approximation of each circuit, the simple feedforward control is sufficient for circuits A, B and C.

The simulation and experimental results demonstrate excellent dynamic performances with resonance being properly damped. This verifies the effectiveness of the VCC method. Additionally, the VCC method can be seamlessly integrated with the current controllers in the literature to remedy the steady-state errors and distortion, e.g. a disturbance observer and a harmonic controller.

\section{APPENDIX}

\section{SOLUtion to THE VCC PROBLEM}

The CT VCC problem in (5), (6) and (7) has an exact solution if $R_{3 \mathrm{v}}^{\mathrm{DM}}=0$. However, to employ the effect of $R_{3 \mathrm{v}}^{\mathrm{DM}}$ (Section VI-B), the solution is relaxed by satisfying (5) and (6), and matching only the low-frequency behavior of the voltage/current control design. The analysis herein is based on the CT state-space model, and also holds for the DT VCC problem in (11). The solution is derived based on the transformation to controllable canonical form [?].

The pair $\left(\mathbf{A}, \mathbf{B}_{\mathrm{i}}\right)$ of a state-space system of order $n$ is controllable if the eigenvalues of $\mathbf{A}-\mathbf{B}_{\mathrm{i}} \mathbf{L}_{\mathrm{xc}}$ can be arbitrarily placed with a constant gain $\mathbf{L}_{\mathrm{xc}}$. The corresponding full-rank controllability matrix is

$$
\mathbf{W}_{\left(\mathbf{A}, \mathbf{B}_{\mathrm{i}}\right)}=\left[\begin{array}{llll}
\mathbf{B}_{\mathrm{i}} & \mathbf{A B}_{\mathrm{i}} & \ldots & \mathbf{A}^{n-1} \mathbf{B}_{\mathrm{i}}
\end{array}\right] .
$$

Additionally, the pair $\left(\mathbf{A}, \mathbf{B}_{\mathrm{i}}\right)$ can be transformed into the controllable canonical form $\mathbf{S}:\left(\mathbf{A}, \mathbf{B}_{\mathrm{i}}\right) \stackrel{\mathrm{z}=\mathbf{S x}}{\longmapsto}\left(\mathbf{S A S}^{-1}, \mathbf{S B}_{\mathrm{i}}\right)$ where

$$
\begin{aligned}
& \mathbf{S}_{\left(\mathbf{A}, \mathbf{B}_{\mathrm{i}}\right)}= \\
& {\left[\begin{array}{llll}
\mathcal{B} & \mathbf{W}^{T} \mathbf{A}^{T} \mathbf{W}^{-T} \mathcal{B} & \ldots & \mathbf{W}^{T}\left(\mathbf{A}^{T}\right)^{n-1} \mathbf{W}^{-T} \mathcal{B}
\end{array}\right] \mathbf{W}^{-1}}
\end{aligned}
$$

with $\mathcal{B}=\left[\begin{array}{llll}0 & \cdots & 0 & 1\end{array}\right]^{T}$ and $\mathbf{W}=\mathbf{W}_{\left(\mathbf{A}, \mathbf{B}_{\mathrm{i}}\right)}$. The gain $\mathbf{L}_{\mathrm{xc}}$ can be computed from

$$
\mathbf{L}_{\mathrm{xc}}=\mathcal{B}\left(\mathbf{S}_{\mathrm{p}} \mathbf{A}_{\mathrm{p}} \mathbf{S}_{\mathrm{p}}^{-1}-\mathbf{S}_{\mathrm{vc}} \mathbf{A}_{\mathrm{vc}} \mathbf{S}_{\mathrm{vc}}^{-1}\right) \mathbf{S}_{\mathrm{p}}
$$

with $\mathbf{S}_{\mathrm{p}}=\mathbf{S}_{\left(\mathbf{A}_{\mathrm{p}}, \mathbf{B}_{\mathrm{i}, \mathrm{p}}\right)}$ and $\mathbf{S}_{\mathrm{vc}}=\mathbf{S}_{\left(\mathbf{A}_{\mathrm{vc}}, \mathbf{B}_{\mathrm{i}, \mathrm{vc}}\right)}$ [?].

Knowing the gain $\mathbf{L}_{\mathrm{xc}}$, a similarity transformation $\mathbf{T}_{\mathrm{c}}$ satisfying (5) and (6) can then be found from [?]:

$$
\frac{1}{L_{\mathrm{uc}}} \mathbf{T}_{\mathrm{c}}=\mathbf{W}_{\mathrm{f}} \mathbf{W}_{\mathrm{vc}}^{-1}
$$

with $\mathbf{A}_{\mathrm{f}}=\mathbf{A}_{\mathrm{p}}-\mathbf{B}_{\mathrm{i}, \mathrm{p}} \mathbf{L}_{\mathrm{xc}}, \mathbf{W}_{\mathrm{f}}=\mathbf{W}_{\left(\mathbf{A}_{\mathrm{f}}, \mathbf{B}_{\mathrm{i}, \mathrm{p}}\right)}, \mathbf{W}_{\mathrm{vc}}=$ $\mathbf{W}_{\left(\mathbf{A}_{\mathrm{vc}}, \mathbf{B}_{\mathrm{i}, \mathrm{vc}}\right)}$. The gains $L_{\mathrm{uc}}$ and $L_{\mathrm{oc}}$ are computed to match the low-frequency behaviors of the physical circuit to the ones of the virtual circuit, i.e. DM grid current and CM capacitor voltage:

$$
\begin{gathered}
L_{\mathrm{uc}}=\left(\mathbf{C}_{\mathrm{o}} \mathbf{N}_{0, \mathrm{f}} \mathbf{B}_{\mathrm{i}, \mathrm{p}}\right)^{-1}\left(\mathbf{C}_{\mathrm{o}} \mathbf{N}_{0, \mathrm{vc}} \mathbf{B}_{\mathrm{i}, \mathrm{vc}}\right) \\
L_{\mathrm{oc}}=\left(\mathbf{C}_{\mathrm{o}} \mathbf{N}_{0, \mathrm{f}} \mathbf{B}_{\mathrm{i}, \mathrm{p}}\right)^{-1}\left(\mathbf{C}_{\mathrm{o}}\left(\mathbf{N}_{0, \mathrm{vc}} \mathbf{B}_{\mathrm{i}, \mathrm{vc}}-\mathbf{N}_{0, \mathrm{f}} \mathbf{B}_{\mathrm{o}, \mathrm{p}}\right)\right)
\end{gathered}
$$

where $\mathbf{N}_{0, x}=\mathbf{A}_{x}^{-1}, x \in\{\mathrm{f}, \mathrm{vc}\}$ and $\mathbf{C}_{\mathrm{o}}$ corresponds to $\mathbf{C}_{\mathrm{o}}^{\mathrm{DM}}=\left[\begin{array}{lll}0 & 0 & 1\end{array}\right]$ and $\mathbf{C}_{\mathrm{o}}^{\mathrm{CM}}=\left[\begin{array}{ll}1 & 0\end{array}\right]$ from (1). 